\title{
ON STABILITY OF THE CRANK-NICOLSON SCHEME WITH APPROXIMATE TRANSPARENT BOUNDARY CONDITIONS FOR THE SCHRÖDINGER EQUATION. PART II*
}

\author{
B. DUCOMET ${ }^{\dagger}$ AND A. ZLOTNIK
}

\begin{abstract}
We continue to consider initial-boundary value problems for a generalized timedependent Schrödinger equation in $1 \mathrm{D}$ on the semi-axis and in 2D on a semi-bounded strip. The Crank-Nicolson finite-difference schemes with general approximate transparent boundary conditions (TBCs), including the discrete TBCs, are investigated. We prove unconditional stability in $L^{2}$ and in the energy norm with respect to initial data and free terms in the equation and the approximate TBC, in general non-uniform in time, under new suitable conditions (inequalities) on a non-local operator $\mathcal{S}$ of the approximate TBC. These inequalities are valid for the operators $\mathcal{S}_{\text {ref }}$ of the discrete TBCs. The results are applied to derive collections of stability bounds with respect to the perturbation $\mathcal{S}_{\text {ref }}-\mathcal{S}$. For the discrete convolution-type operators $\mathcal{S}$ (in particular, for $\mathcal{S}_{\text {ref }}$ ), we present necessary and sufficient conditions for the stability inequalities to be valid, together with bounds for norms of $\mathcal{S}_{\text {ref }}-\mathcal{S}$, in terms of reproducing functions of the convolution kernels. The analysis exploits the Hardy spaces of analytic functions.
\end{abstract}

Key words. Stability, Crank-Nicolson finite-difference scheme, transparent boundary conditions, time-dependent Schrödinger equation, discrete convolution operator, Hardy spaces

AMS subject classifications. $\quad 65 \mathrm{M} 06,65 \mathrm{M} 12,35 \mathrm{Q} 40$

\section{Introduction}

We continue to consider initial-boundary value problems for a generalized timedependent Schrödinger equation in 1D on the semi-axis and in 2D on a semi-bounded strip closely related to a microscopic description of low-energy nuclear fission dynamics $[7,13]$. We investigate the Crank-Nicolson finite-difference schemes with general approximate transparent boundary conditions (TBCs) including the discrete TBCs $[4,5,12]$.

The importance of the stability problem is well-known for mesh methods with the approximate TBCs of solving time-dependent Schrödinger-like equations in unbounded domains see [1]-[6], [8, 9, 12], [15]-[17], [19]-[21]. In Part I of this study (see $[10]$ ), a new form of the approximate TBCs has been suggested, with a non-local operator $\mathcal{S}$ governing properties of the schemes. The uniform-in-time stability bounds in $L^{2}$ have been proved under suitable condition (inequality) on $\mathcal{S}$, for non-uniform meshes in space and time.

In the present Part II, we establish essential further results in this direction. We prove unconditional stability in $L^{2}$ and in the energy norm with respect to initial data and free terms in the equation and the approximate TBC, in general non-uniform in time in order to cover broader applications. To this end, we introduce new suitable conditions (inequalities) on $\mathcal{S}$. These inequalities are valid for the operators $\mathcal{S}_{\text {ref }}$ of the discrete TBCs (ensuring the uniform-in-time stability); we clarify them by considering the corresponding schemes on infinite space meshes. We suggest a trick reducing the derivation of general non-uniform in time bounds to the derivation of simpler uniform

${ }^{*}$ Received: October 22, 2006; accepted (in revised version): February 8, 2007. Communicated by Francois Golse.

${ }^{\dagger}$ DPTA/Service de Physique Nucléaire, CEA/DAM Ile de France, BP 12, F-91680 Bruyères-leChâtel, France (bernard.ducomet@cea.fr).

${ }^{\ddagger}$ Department of Applied Mathematics, Russian State Social University, W. Pieck 4, 129226 Moscow, Russia (zlotnik@apmsun.mpei.ac.ru). 
ones. Note that our bounds in the energy norm do not impose additional restrictions on the mesh Hamiltonian operators, in particular, on their potentials.

These stability bounds are applied to derive collections of stability bounds with respect to the perturbation $\mathcal{S}_{\text {ref }}-\mathcal{S}$, both non-uniform and uniform with respect to the space mesh step; the bounds in the energy norm are exploited to prove the latter stability bounds. These collections of bounds are important for construction and analysis of the simplified discrete TBCs [5].

In the 1D case, for the operators $\mathcal{S}$ of a discrete convolution type (in particular, for $\mathcal{S}_{\text {ref }}$ ) and the uniform time mesh, we present necessary and sufficient conditions for validity of the stability inequalities in terms of inequalities for reproducing functions $q$ of the convolution kernels. The latter inequalities hold for the reproducing function $q_{\mathrm{ref}}$ corresponding to $\mathcal{S}_{\text {ref }}$. Moreover, using a slightly different discrete convolution representation (with improved properties of the convolution kernel), we present bounds for norms of $\mathcal{S}_{\text {ref }}-\mathcal{S}$ by the $L^{s}$-norms of $q_{\mathrm{ref}}-q$, both non-uniform (for $1 \leqslant s<\infty$ ) and uniform (for $s=\infty$ ) with respect to the time mesh step. It is natural to exploit the classical Hardy spaces of analytic functions in this analysis.

In the 2D case, we also introduce an important class of the approximate TBCs (including the discrete TBC) allowing to reduce both the 2D stability inequalities and the 2D bounds for norms of $\mathcal{S}_{\text {ref }}-\mathcal{S}$ to their 1D counterparts.

Our results essentially develop the corresponding stability analysis in [5]. In the short form they are partially presented in [23].

The paper is organized as follows. Section 2 is devoted to the stability study in the 1D case for general $\mathcal{S}$ and for $\mathcal{S}_{\text {ref }}$. In Section 3, the case of the 1D discrete convolution-type operators $\mathcal{S}$ is considered. Section 4 deals with the stability study in the $2 \mathrm{D}$ case.

2. Stability bounds for the $1 D$ Crank-Nicolson scheme with an approximate TBC

We first consider the generalized 1D time-dependent Schrödinger equation

$$
i \hbar \frac{\partial \psi}{\partial t}=\mathcal{H} \psi:=-\frac{\hbar^{2}}{2} \frac{\partial}{\partial x}\left(B \frac{\partial \psi}{\partial x}\right)+V \psi, \text { for } x>0 \text { and } t>0
$$

involving the 1D Hamiltonian operator $\mathcal{H}$, for the unknown complex-valued wave function $\psi=\psi(x, t)$. Hereafter $i$ is the imaginary unit, $\hbar=$ const $>0$ and $B=B(x) \geqslant$ $\nu>0$ and $V=V(x)$ are given real-valued functions.

Equation (2.1) is supplemented with the following boundary condition, the condition at infinity and the initial condition

$$
\begin{aligned}
& \left.\psi\right|_{x=0}=0 \text { and } \psi(x, t) \rightarrow 0 \text { as } x \rightarrow \infty, \text { for any } t>0, \\
& \left.\psi\right|_{t=0}=\psi^{0}(x) \text { for } x>0 .
\end{aligned}
$$

We assume that, for some $X_{0}>0, B(x)=B_{1 \infty}>0, V(x)=V_{\infty}$ and $\psi^{0}(x)=0$ for any $x \geqslant X_{0}$.

We write down an integro-differential TBC for this problem in the form, for any $X \geqslant X_{0}$ (for example see [12])

$$
\frac{\partial \psi}{\partial x}(X, t)=-\frac{1-i}{\sqrt{\hbar B_{1 \infty}}} e^{-i\left(V_{\infty} / \hbar\right) t} \frac{1}{\sqrt{\pi}} \frac{d}{d t} \int_{0}^{t} \psi(X, \theta) e^{i\left(V_{\infty} / \hbar\right) \theta} \frac{d \theta}{\sqrt{t-\theta}} \text { for } t>0 .
$$


We need to recall and to extend some notations from Part I. We fix some $X>$ $X_{0}$ and introduce a non-uniform mesh $\bar{\omega}_{h, \infty}$ in $x$ on $[0, \infty)$ with the nodes $0=x_{0}<$ $\cdots<x_{J}=X<\ldots$ and the steps $h_{j}:=x_{j}-x_{j-1}$ such that $h_{J} \leqslant X-X_{0}$ and $h_{j}=h \equiv$ $h_{J}$ for $j \geqslant J$. We also introduce a non-uniform mesh $\bar{\omega}^{\tau}$ in $t$ on $[0, \infty)$ with the nodes $0=t_{0}<\cdots<t_{m}<\ldots, t_{m} \rightarrow \infty$ as $m \rightarrow \infty$, and the steps $\tau_{m}:=t_{m}-t_{m-1}$. Let $\omega_{h, \infty}:=\bar{\omega}_{h, \infty} \backslash\{0\}, \bar{\omega}_{h}:=\left\{x_{j}\right\}_{j=0}^{J}, \omega_{h}:=\left\{x_{j}\right\}_{j=1}^{J-1}$ and $\omega^{\tau}:=\bar{\omega}^{\tau} \backslash\{0\}, \omega_{M}^{\tau}:=\left\{t_{m}\right\}_{m=1}^{M}$ and $\bar{\omega}_{M}^{\tau}:=\left\{t_{m}\right\}_{m=0}^{M}$.

We define the backward, the modified forward and the central difference quotients with respect to $x$

$$
\bar{\partial}_{x} W_{j}:=\frac{W_{j}-W_{j-1}}{h_{j}}, \widehat{\partial}_{x} W_{j}:=\frac{W_{j+1}-W_{j}}{h_{j+1 / 2}}, \stackrel{\circ}{\partial_{x}} W_{j}:=\frac{W_{j+1}-W_{j-1}}{2 h_{j+1 / 2}},
$$

where $h_{j+1 / 2}:=\left(h_{j}+h_{j+1}\right) / 2$, together with the backward difference quotient, an averaging and the backward shift in time

$$
\bar{\partial}_{t} \Phi^{m}:=\frac{\Phi^{m}-\Phi^{m-1}}{\tau_{m}}, \quad \bar{s}_{t} \Phi^{m}:=\frac{\Phi^{m-1}+\Phi^{m}}{2}, \quad \check{\Phi}^{m}:=\Phi^{m-1} .
$$

We introduce some mesh counterparts of the inner product in the complex spaces $L^{2}(0, X)$ and $L^{2}\left(0, t_{M}\right)$ :

$$
\begin{aligned}
(U, W)_{\omega_{h}}:=\sum_{j=1}^{J-1} U_{j} W_{j}^{*} h_{j+1 / 2}, \quad(U, W)_{\bar{\omega}_{h}}: & =(U, W)_{\omega_{h}}+U_{J} W_{J}^{*} \frac{h}{2}, \\
(U, W)_{\widetilde{\omega}_{h}}: & =\sum_{j=1}^{J} U_{j} W_{j}^{*} h_{j}, \\
(\Phi, Y)_{\omega_{M}^{\tau}}: & =\sum_{m=1}^{M} \Phi^{m}\left(Y^{m}\right)^{*} \tau_{m},
\end{aligned}
$$

together with the associated mesh norms $\|\cdot\|_{\omega_{h}},\|\cdot\|_{\bar{\omega}_{h}},\|\cdot\|_{\widetilde{\omega}_{h}}$ and $\|\cdot\|_{\omega_{M}^{\tau}}$. Hereafter $z^{*}, \operatorname{Re} z$ and $\operatorname{Im} z$ denote the complex conjugate, the real and the imaginary parts of $z \in \mathbb{C}$.

We also use the 1D mesh Hamiltonian operator

$$
\mathcal{H}_{h} W:=-\frac{\hbar^{2}}{2} \widehat{\partial}_{x}\left(B_{h} \bar{\partial}_{x} W\right)+V_{h} W
$$

where $B_{h j}=B\left(x_{j}-h_{j} / 2\right)$ and $V_{h j}=V\left(x_{j}\right)$ (for definiteness), and we study the CrankNicolson scheme with general approximate TBC of the form from Part I

$$
\begin{gathered}
i \hbar \bar{\partial}_{t} \Psi=\mathcal{H}_{h} \bar{s}_{t} \Psi+F \text { on } \omega_{h} \times \omega^{\tau}, \\
\Psi_{0}^{m}=0 \text { for } m \geqslant 1, \\
\mathcal{D}_{\Gamma} \Psi_{J}^{m}:=\left[\frac{\hbar^{2}}{2} B_{1 \infty} \bar{\partial}_{x} \bar{s}_{t} \Psi-\frac{h}{2}\left(i \hbar \bar{\partial}_{t} \Psi-V_{\infty} \bar{s}_{t} \Psi\right)\right]_{J}^{m}
\end{gathered}
$$




$$
\begin{gathered}
=\frac{\hbar^{2}}{2} B_{1 \infty} \mathcal{S}^{m} \Psi_{J}^{m}+\hat{G}^{m} \text { for } m \geqslant 1 \\
\Psi^{0}=\Psi_{h}^{0} \text { on } \bar{\omega}_{h}
\end{gathered}
$$

where $\Psi_{h_{j}}^{0}=\psi^{0}\left(x_{j}\right)$ (for definiteness) and thus $\Psi_{h J}^{0}=0$, and the condition $\Psi_{h_{0}}^{0}=0$ is also assumed. Moreover, $\Psi_{J}^{m}=\left\{\Psi_{J}^{n}\right\}_{n=1}^{m}$, and $\mathcal{S} \boldsymbol{\Psi}_{J}$ serves as general (abstract) approximation of the right-hand side in the TBC (2.4); a discussion of this form of the approximation is given in Part I. We do not suppose that the operator $\mathcal{S}$ is linear. Finally, $F: \omega_{h} \times \omega^{\tau} \rightarrow \mathbb{C}$ and $\hat{G}: \omega^{\tau} \rightarrow \mathbb{C}$ are given perturbations that are introduced to study the stability of the scheme in more detail.

We define the class $N D\left(\bar{\omega}^{\tau}\right)$ of non-decreasing functions $E$ on $\bar{\omega}^{\tau}$ such that $E^{0}=1$. This allows us to cover a broader family of approximate TBCs.

Proposition 2.1. Let $\Psi$ be a solution of the finite-difference scheme (2.5)-(2.8) with any $\Psi_{h}^{0}$ such that $\Psi_{h 0}^{0}=\Psi_{h J}^{0}=0$. Assume that the operator $\mathcal{S}$ satisfies the inequality

$$
\operatorname{Im}\left(\frac{1}{E^{2}} \mathcal{S} \Phi, \bar{s}_{t} \Phi\right)_{\omega_{M}^{\tau}} \geqslant 0 \text { for any } M \geqslant 1
$$

for some $E \in N D\left(\bar{\omega}^{\tau}\right)$ and any function $\Phi: \bar{\omega}^{\tau} \rightarrow \mathbb{C}$ such that $\Phi^{0}=0$. Then the following stability bound holds, for any $M \geqslant 1$

$$
\begin{gathered}
\max _{0 \leqslant m \leqslant M}\left\|\Psi^{m}\right\|_{\bar{\omega}_{h}} \\
\leqslant E^{M}\left(\left\|\Psi_{h}^{0}\right\|_{\bar{\omega}_{h}}+\frac{2}{\hbar} \sum_{m=1}^{M} \frac{1}{E^{m}}\left\|F^{m}\right\|_{\omega_{h}} \tau_{m}+\frac{2}{\hbar} \sqrt{\frac{2}{h}} \sum_{m=1}^{M} \frac{1}{E^{m}}\left|\hat{G}^{m}\right| \tau_{m}\right) .
\end{gathered}
$$

Proof. We recall first the energy-type equality from the proof of the similar Proposition 2.1 in Part I

$$
\frac{\hbar}{2} \bar{\partial}_{t}\|\Psi\|_{\bar{\omega}_{h}}^{2}=\operatorname{Im}\left\{\left(F, \bar{s}_{t} \Psi\right)_{\omega_{h}}-\hat{G}\left(\bar{s}_{t} \Psi_{J}\right)^{*}-\frac{\hbar^{2}}{2} B_{1 \infty} \mathcal{S} \Psi_{J}\left(\bar{s}_{t} \Psi_{J}\right)^{*}\right\} .
$$

Substituting $\Psi=E Y$ and dividing the result by $E^{2}$, we get the following relations for the auxiliary function $Y$ :

$$
\begin{gathered}
\frac{\hbar}{2}\left[\bar{\partial}_{t}\|Y\|_{\bar{\omega}_{h}}^{2}+\frac{\bar{\partial}_{t}\left(E^{2}\right)}{E^{2}}\|\check{Y}\|_{\bar{\omega}_{h}}^{2}\right] \\
=\operatorname{Im}\left\{\left(F, \frac{1}{E^{2}} \bar{s}_{t}(E Y)\right)_{\omega_{h}}-\hat{G} \frac{1}{E^{2}}\left(\bar{s}_{t}\left(E Y_{J}\right)\right)^{*}-\frac{\hbar^{2}}{2 E^{2}} B_{1 \infty} \mathcal{S} \Psi_{J}\left(\bar{s}_{t} \Psi_{J}\right)^{*}\right\} \\
\leqslant\|F\|_{\omega_{h}} \frac{1}{E} \bar{s}_{t}\|Y\|_{\omega_{h}}+|\hat{G}| \frac{1}{E} \bar{s}_{t}\left|Y_{J}\right|-\frac{\hbar^{2}}{2 E^{2}} B_{1 \infty} \operatorname{Im}\left(\mathcal{S} \Psi_{J}\left(\bar{s}_{t} \Psi_{J}\right)^{*}\right) .
\end{gathered}
$$


The standard argument involving the multiplication by $2 \tau / \hbar$, the summation over $m=1, \ldots, M$ together with the application of the inequality $\bar{\partial}_{t} E \geqslant 0$ and condition (2.9) lead us to the following bound for $Y$ :

$$
\max _{0 \leqslant m \leqslant M}\left\|Y^{m}\right\|_{\bar{\omega}_{h}} \leqslant\left\|Y^{0}\right\|_{\bar{\omega}_{h}}+\frac{2}{\hbar} \sum_{m=1}^{M} \frac{1}{E^{m}}\left\|F^{m}\right\|_{\omega_{h}} \tau_{m}+\frac{2}{\hbar} \sqrt{\frac{2}{h}} \sum_{m=1}^{M} \frac{1}{E^{m}}\left|\hat{G}^{m}\right| \tau_{m},
$$

for any $M \geqslant 1$. The inverse substitution $Y=\Psi / E$ clearly implies the result.

REMARK 2.1. It is easy to check that the quantity

$E^{M}\left[\hbar B_{1 \infty} \operatorname{Im}\left(\frac{1}{E^{2}} \mathcal{S} \Psi_{J}, \bar{s}_{t} \Psi_{J}\right)_{\omega_{M}^{\tau}}\right]^{1 / 2}$ is also bounded by the right-hand side of (2.10), for any $M \geqslant 1$.

Corollary 2.2. Let the hypotheses of Proposition 2.1 together with the a priori estimate

$$
\left\|\bar{\partial}_{x} \Psi\right\|_{\widetilde{\omega}_{h}} \leqslant C_{0} \hat{E} \quad \text { on } \bar{\omega}^{\tau}
$$

for some $\hat{E} \in N D\left(\bar{\omega}^{\tau}\right)$, be valid. Then the $\hat{G}$-term in bound (2.10) can be replaced by

$$
\left[\frac{2 \sqrt{2 C_{0}}}{\hbar} \sum_{m=1}^{M} \frac{\sqrt{\bar{s}_{t} \hat{E}^{m}}}{E^{m}}\left|\hat{G}^{m}\right| \tau_{m}\right]^{2 / 3}
$$

Proof. According to the previous proof, for $\bar{Y}^{M}:=\max _{0 \leqslant m \leqslant M}\left\|Y^{m}\right\|_{\bar{\omega}_{h}}$, the following inequality

$$
\left(\bar{Y}^{M}\right)^{2} \leqslant\left\|Y^{0}\right\|_{\bar{\omega}_{h}}^{2}+\left(\frac{2}{\hbar} \sum_{m=1}^{M} \frac{1}{E^{m}}\left\|F^{m}\right\|_{\omega_{h}} \tau_{m}\right) \bar{Y}^{M}+\frac{2}{\hbar}\left(|\hat{G}|, \frac{1}{E} \bar{s}_{t}\left|Y_{J}\right|\right)_{\omega_{M}^{\tau}},
$$

holds. (Actually, (2.13) follows from this one.) Using the well-known multiplicative inequality

$$
\|W\|_{C\left(\bar{\omega}_{h}\right)}^{2}:=\max _{0 \leqslant j \leqslant J}\left|W_{j}\right|^{2} \leqslant 2\|W\|_{\bar{\omega}_{h}}\left\|\bar{\partial}_{x} W\right\|_{\widetilde{\omega}_{h}}
$$

(with $W_{0}=0$ ), together with the a priori estimate (2.14), we get

$$
\frac{2}{\hbar}\left(|\hat{G}|, \frac{1}{E} \bar{s}_{t}\left|Y_{J}\right|\right)_{\omega_{M}^{\tau}} \leqslant \sqrt{2} C_{0} \frac{2}{\hbar}\left(|\hat{G}|, \frac{1}{E} \sqrt{\bar{s}_{t} \hat{E}}\right)_{\omega_{M}^{\tau}} \sqrt{\bar{Y}^{M}} .
$$

Plugging this bound into the previous inequality for $\bar{Y}^{M}$ and solving the arising elementary quartic inequality for $\sqrt{\bar{Y}^{M}}$, we find that the $\hat{G}$-term in (2.13) can be replaced by

$$
\left[\sqrt{2 C_{0}} \frac{2}{\hbar}\left(|\hat{G}|, \frac{1}{E} \sqrt{\bar{s}_{t} \hat{E}}\right)_{\omega_{M}^{\tau}}\right]^{2 / 3},
$$

which implies the result. 
Note that the new term $(2.15)$ is a norm of $\hat{G}$ to the power $2 / 3$ only, but no longer contains the factor $\sqrt{2 / h}$.

Obviously, any function $E \in N D\left(\bar{\omega}^{\tau}\right)$ may be written as $E^{M}=\prod_{1 \leqslant m \leqslant M} e_{m}$ with $e_{m}:=E^{m} / E^{m-1} \geqslant 1$. If $e_{m} \leqslant 1+c_{0} \tau_{m}$ for any $m \geqslant 1$, for some $c_{0} \geqslant 0$, then $E^{M} / E^{m} \leqslant$ $e^{c_{0}\left(t_{M}-t_{m}\right)}$ for any $0 \leqslant m \leqslant M$.

We define the Hermitian-symmetric bilinear form,

$$
\mathcal{L}_{\bar{\omega}_{h}}(U, W):=\frac{\hbar^{2}}{2}\left(B_{h} \bar{\partial}_{x} U, \bar{\partial}_{x} W\right)_{\widetilde{\omega}_{h}}+(V U, W)_{\bar{\omega}_{h}}
$$

on functions $U, W: \bar{\omega}_{h} \rightarrow \mathbb{C}$. The identity

$$
\mathcal{L}_{\bar{\omega}_{h}}(U, W)=\left(\mathcal{H}_{h} U, W\right)_{\omega_{h}}+\left(\frac{\hbar^{2}}{2} B_{1 \infty} \bar{\partial}_{x} U_{J}+\frac{h}{2} V_{\infty} U_{J}\right) W_{J}^{*}
$$

holds provided that $W_{0}=0$. For any such $W$, one has clearly

$$
\|W\|_{\mathcal{H}_{h}+v I ; \bar{\omega}_{h}}^{2}:=\mathcal{L}_{\bar{\omega}_{h}}(W, W)+v\|W\|_{\bar{\omega}_{h}}^{2} \geqslant \nu_{1}\left\|\bar{\partial}_{x} W\right\|_{\widetilde{\omega}_{h}}^{2}
$$

with $\nu_{1}:=\nu \hbar^{2} / 2$, for any real number $v \geqslant v_{0}$. Here $v_{0}:=-\min _{1 \leqslant j \leqslant J} V_{j}$; in particular, $v_{0} \leqslant 0$ provided that $V \geqslant 0$. $I$ is the identity operator.

Proposition 2.3. Let $\Psi$ be a solution of the finite-difference scheme (2.5)-(2.8) with any $\Psi_{h}^{0}$ such that $\Psi_{h 0}^{0}=\Psi_{h J}^{0}=0$ and $\hat{G}=0$. Assume that the operator $\mathcal{S}$ satisfies the inequality

$$
\operatorname{Im}\left(\mathcal{S} \Phi, i \hbar \bar{\partial}_{t} \Phi+v \bar{s}_{t} \Phi\right)_{\omega_{M}^{\tau}} \geqslant 0 \text { for any } M \geqslant 1
$$

for any $\Phi: \bar{\omega}^{\tau} \rightarrow \mathbb{C}$ such that $\Phi^{0}=0$ and some $v \geqslant v_{0}$. Then the following bound holds:

$$
\max _{0 \leqslant m \leqslant M}\left\|\Psi^{m}\right\|_{\mathcal{H}_{h}+v I ; \bar{\omega}_{h}} \leqslant\left\|\Psi_{h}^{0}\right\|_{\mathcal{H}_{h}+v I ; \bar{\omega}_{h}}+\frac{2}{\hbar} \sum_{m=1}^{M}\left\|F^{m}\right\|_{\mathcal{H}_{h}+v I ; \bar{\omega}_{h}} \tau_{m} \text { for any } M \geqslant 1,
$$

where $F^{m}$ is extended by $F_{0}^{m}=F_{J}^{m}=0$.

Proof. Taking the $(\cdot, \cdot)_{\omega_{h}}$-inner product of equation $(2.5)$ and $\mathcal{H}_{h} \bar{s}_{t} \Psi$ and applying identity (2.17), we get

$$
\begin{gathered}
\mathcal{L}_{\bar{\omega}_{h}}\left(i \hbar \bar{\partial}_{t} \Psi, \bar{s}_{t} \Psi\right)-i \hbar \bar{\partial}_{t} \Psi_{J}\left(\frac{\hbar^{2}}{2} B_{1 \infty} \bar{\partial}_{x} \bar{s}_{t} \Psi_{J}+\frac{h}{2} V_{\infty} \bar{s}_{t} \Psi_{J}\right)^{*} \\
=\left\|\mathcal{H}_{h} \bar{s}_{t} \Psi\right\|_{\omega_{h}}^{2}+\left(F, \mathcal{H}_{h} \bar{s}_{t} \Psi\right)_{\omega_{h}} .
\end{gathered}
$$

Since according to the boundary condition (2.7) for $\hat{G}=0$

$$
\frac{\hbar^{2}}{2} B_{1 \infty} \bar{\partial}_{x} \bar{s}_{t} \Psi_{J}+\frac{h}{2} V_{\infty} \bar{s}_{t} \Psi_{J}=\frac{h}{2} i \hbar \bar{\partial}_{t} \Psi_{J}+\frac{\hbar^{2}}{2} B_{1 \infty} \mathcal{S} \Psi_{J},
$$

taking the imaginary part, we have

$$
\frac{\hbar}{2} \bar{\partial}_{t} \mathcal{L}_{\bar{\omega}_{h}}(\Psi, \Psi)=\operatorname{Im}\left\{\left(F, \mathcal{H}_{h} \bar{s}_{t} \Psi\right)_{\omega_{h}}-\frac{\hbar^{2}}{2} B_{1 \infty}\left(\mathcal{S} \Psi_{J}\right)\left(i \hbar \bar{\partial}_{t} \Psi_{J}\right)^{*}\right\} .
$$


Combining this equality with the corresponding equality (2.11) from the previous proof, we obtain

$$
\begin{gathered}
\frac{\hbar}{2} \bar{\partial}_{t}\left[\mathcal{L}_{\bar{\omega}_{h}}(\Psi, \Psi)+v\|\Psi\|_{\bar{\omega}_{h}}^{2}\right] \\
=\operatorname{Im}\left\{\left(F,\left(\mathcal{H}_{h}+v I\right) \bar{s}_{t} \Psi\right)_{\omega_{h}}-\frac{\hbar^{2}}{2} B_{1 \infty}\left(\mathcal{S} \Psi_{J}\right)\left(i \hbar \bar{\partial}_{t} \Psi_{J}+v \bar{s}_{t} \Psi\right)^{*}\right\} .
\end{gathered}
$$

Once again multiplying this by $2 \tau / \hbar$, summing over $m=1, \ldots, M$ and applying (2.18) and (2.19), we get the relations

$$
\begin{aligned}
\left\|\Psi^{M}\right\|_{\mathcal{H}_{h}+v I ; \bar{\omega}_{h}}^{2} & \leqslant\left\|\Psi^{0}\right\|_{\mathcal{H}_{h}+v I ; \bar{\omega}_{h}}^{2}+\frac{2}{\hbar} \operatorname{Im} \sum_{m=1}^{M}\left(F^{m},\left(\mathcal{H}_{h}+v I\right) \bar{s}_{t} \Psi^{m}\right)_{\omega_{h}} \tau_{m} \\
& \leqslant\left\|\Psi^{0}\right\|_{\mathcal{H}_{h}+v I ; \bar{\omega}_{h}}^{2}+\frac{2}{\hbar} \sum_{m=1}^{M}\left\|F^{m}\right\|_{\mathcal{H}_{h}+v I ; \bar{\omega}_{h}}\left(\bar{s}_{t}\|\Psi\|_{\mathcal{H}_{h}+v I ; \bar{\omega}_{h}}\right)^{m} \tau_{m}
\end{aligned}
$$

which yield the result.

Corollary 2.4. Let us consider the scheme (2.5)-(2.8) in the case $F=0$ and $\hat{G}=0$, for some $v \geqslant v_{0}$. If condition (2.19) is valid for $\Phi=\Psi_{J}$, then the solution satisfies the equality

$$
\max _{m \geqslant 0}\left\|\Psi^{m}\right\|_{\mathcal{H}_{h}+v I ; \bar{\omega}_{h}}=\left\|\Psi_{h}^{0}\right\|_{\mathcal{H}_{h}+v I ; \bar{\omega}_{h}} .
$$

Conversely, if this equality is satisfied, then condition (2.19) for $\Phi=\Psi_{J}$ is valid.

Proof. Equality (2.21) for $F=0$ implies that

$$
\left\|\Psi^{M}\right\|_{\mathcal{H}_{h}+v I ; \bar{\omega}_{h}}^{2}-\left\|\Psi^{0}\right\|_{\mathcal{H}_{h}+v I ; \bar{\omega}_{h}}^{2}=-\hbar B_{1 \infty} \operatorname{Im}\left(\mathcal{S} \Psi_{J}, i \hbar \bar{\partial}_{t} \Psi_{J}+v \bar{s}_{t} \Psi_{J}\right)_{\omega_{M}^{\tau}},
$$

for any $M \geqslant 1$, and the result follows.

A similar property relating $\left\|\Psi^{m}\right\|_{\bar{\omega}_{h}}$ to condition (2.9) for $E \equiv 1$ and $\Phi=\Psi_{J}$ is contained in Part I. Note that condition (2.9) in Proposition 2.1 and condition (2.19) in Proposition 2.3 have been applied for $\Phi=\Psi_{J}$ only.

Now we analyze and prove condition (2.19) in the case of the discrete TBC. To this end, we turn to the Crank-Nicolson scheme on the infinite space mesh for the original problem (2.1)-(2.3)

$$
\begin{aligned}
i \hbar \bar{\partial}_{t} \Psi & =\mathcal{H}_{h} \bar{s}_{t} \Psi+F \text { on } \omega_{h, \infty} \times \omega^{\tau}, \\
\Psi_{0}^{m} & =0 \text { for } m \geqslant 1, \\
\Psi^{0} & =\Psi_{h}^{0} \text { on } \bar{\omega}_{h, \infty},
\end{aligned}
$$

where $F$ is a given perturbation; this has already been exploited in Part I. We consider only the solutions having the property $\Psi^{m} \in H_{h}$ for any $m \geqslant 0$, where $H_{h}$ is a Hilbert space consisting of mesh functions $W: \bar{\omega}_{h, \infty} \rightarrow \mathbb{C}$ such that $\sum_{j=1}^{\infty}\left|W_{j}\right|^{2}<\infty$ and $W_{0}=0$, equipped with the inner product $(U, W)_{H_{h}}:=\sum_{j=1}^{\infty} U_{j} W_{j}^{*} h_{j+1 / 2}$. 
For $U, W \in H_{h}$ we define the Hermitian-symmetric bilinear form

$$
\mathcal{L}_{\bar{\omega}_{h, \infty}}(U, W):=\frac{\hbar^{2}}{2} \sum_{j=1}^{\infty} B_{h_{j}}\left(\bar{\partial}_{x} U_{j}\right)\left(\bar{\partial}_{x} W_{j}\right)^{*} h_{j}+(V U, W)_{H_{h}}=\left(\mathcal{H}_{h} U, W\right)_{H_{h}}
$$

with the extension $\left(\mathcal{H}_{h} U\right)_{0}:=0$; the equality follows from an identity like $(2.17)$ but for the mesh $\left\{x_{j}\right\}_{j=0}^{j_{0}}$ by passing to the limit as $j_{0} \rightarrow \infty$. Clearly, one has

$$
\|W\|_{\mathcal{H}_{h}+v I}^{2}:=\mathcal{L}_{\bar{\omega}_{h, \infty}}(W, W)+v\|W\|_{H_{h}}^{2} \geqslant \nu_{1} \sum_{j=1}^{\infty}\left|\bar{\partial}_{x} W_{j}\right|^{2} h_{j} \text { for any } W \in H_{h},
$$

for any $v \geqslant v_{0}$.

Proposition 2.5. Let $F^{m} \in H_{h}$ for any $m \geqslant 1$ and $\Psi_{h}^{0} \in H_{h}$. There exists a unique solution of the scheme (2.22)-(2.24), and the following stability bound holds:

$$
\max _{0 \leqslant m \leqslant M}\left\|\Psi^{m}\right\|_{\mathcal{H}_{h}+v I} \leqslant\left\|\Psi_{h}^{0}\right\|_{\mathcal{H}_{h}+v I}+\frac{2}{\hbar} \sum_{m=1}^{M}\left\|F^{m}\right\|_{\mathcal{H}_{h}+v I} \tau_{m} \quad \text { for any } M \geqslant 1,
$$

for any $v \geqslant v_{0}$. Moreover, for $F=0$, the following conservation law holds:

$$
\left\|\Psi^{m}\right\|_{\mathcal{H}_{h}+v I}=\left\|\Psi_{h}^{0}\right\|_{\mathcal{H}_{h}+v I} \text { for any } m \geqslant 0
$$

Proof. The existence and uniqueness of the solution and the boundedness of the operator $\mathcal{H}_{h}: H_{h} \rightarrow H_{h}$, together with the energy-type equality

$$
\frac{\hbar}{2} \bar{\partial}_{t}\|\Psi\|_{H_{h}}^{2}=\operatorname{Im}\left(F, \bar{s}_{t} \Psi\right)_{H_{h}},
$$

are known from Proposition 3.1 in Part I.

Taking the $(\cdot, \cdot)_{H_{h}}$-inner product of equation (2.22) and $\mathcal{H}_{h} \bar{s}_{t} \Psi$, applying identity (2.25) and taking the imaginary part, we get

$$
\frac{\hbar}{2} \bar{\partial}_{t} \mathcal{L}_{\bar{\omega}_{h, \infty}}(\Psi, \Psi)=\operatorname{Im}\left(F, \mathcal{H}_{h} \bar{s}_{t} \Psi\right)_{H_{h}} .
$$

Combining the both equalities, we obtain

$$
\frac{\hbar}{2} \bar{\partial}_{t}\left[\mathcal{L}_{\bar{\omega}_{h, \infty}}(\Psi, \Psi)+v\|\Psi\|_{H_{h}}^{2}\right]=\operatorname{Im}\left(F,\left(\mathcal{H}_{h}+v I\right) \bar{s}_{t} \Psi\right)_{H_{h}} .
$$

The proof is completed in the same manner as the proof of Proposition 2.3.

Corollary 2.6. Let $F_{j}^{m}=0$ and $\Psi_{h j}^{0}=0$ for $j \geqslant J$ and $m \geqslant 1$. If the solution of the scheme (2.22)-(2.24) satisfies the relation

$$
\left(\stackrel{\circ}{\partial}_{x} \bar{s}_{t} \Psi\right)_{J}^{m}=\mathcal{S}_{\mathrm{ref}}^{m} \Psi_{J}^{m} \text { for any } m \geqslant 1
$$

then the following equality holds, for any $M \geqslant 1$ and any real $v$ :

$$
\hbar B_{1 \infty} \operatorname{Im}\left(\mathcal{S}_{\mathrm{ref}} \boldsymbol{\Psi}_{J}, i \hbar \bar{\partial}_{t} \Psi_{J}+v \bar{s}_{t} \Psi_{J}\right)_{\omega_{M}^{\tau}}
$$




$$
=\mathcal{L}_{\bar{\omega}_{h, \infty} \backslash \bar{\omega}_{h}}\left(\Psi^{M}, \Psi^{M}\right)+v\left\|\Psi^{M}\right\|_{\omega_{h, \infty} \backslash \omega_{h}}^{2}
$$

where

$$
\begin{aligned}
\mathcal{L}_{\bar{\omega}_{h, \infty} \backslash \bar{\omega}_{h}}(W, W) & :=\frac{\hbar^{2}}{2} B_{1 \infty} \sum_{j=J+1}^{\infty}\left|\bar{\partial}_{x} W_{j}\right|^{2} h+V_{\infty}\|W\|_{\omega_{h, \infty} \backslash \omega_{h}}^{2} \\
\|W\|_{\omega_{h, \infty} \backslash \omega_{h}}^{2} & :=\frac{h}{2}\left|W_{j}\right|^{2}+\sum_{j=J+1}^{\infty}\left|W_{j}\right|^{2} h
\end{aligned}
$$

Consequently, $\mathcal{S}=\mathcal{S}_{\text {ref }}$ satisfies condition (2.19) for $\Phi=\Psi_{J}$ and any $v \geqslant-V_{\infty}$. Moreover, for the uniform mesh $\bar{\omega}^{\tau}, \mathcal{S}_{\text {ref }}$ is the operator of the $1 D$ discrete TBC, (see [12] and Part I) and the condition is valid for any $\Phi$ mentioned in it (rather than $\Phi=\Psi_{J}$ only).

Proof. Since the solution of (2.22)-(2.24) satisfying (2.28) solves the scheme (2.5)(2.8) with $\hat{G}=0$ too (see the similar Corollary 3.3 in Part I), by subtracting equalities (2.27) and (2.21), we obtain the equality

$$
\frac{\hbar}{2} \bar{\partial}_{t}\left[\mathcal{L}_{\bar{\omega}_{h, \infty} \backslash \bar{\omega}_{h}}(\Psi, \Psi)+v\|\Psi\|_{\omega_{h, \infty} \backslash \omega_{h}}^{2}\right]=\frac{\hbar}{2} B_{1 \infty} \operatorname{Im}\left(\left(\mathcal{S}_{\mathrm{ref}} \boldsymbol{\Psi}_{J}\right)\left(i \hbar \bar{\partial}_{t} \Psi_{J}+v \bar{s}_{t} \Psi_{J}\right)^{*}\right),
$$

which clearly yields equality (2.29).

For the uniform mesh $\bar{\omega}^{\tau}$, it has been proved in Part I (see the first proof of Proposition 3.3) that any function $\Phi: \omega_{M}^{\tau} \rightarrow \mathbb{C}$ can be extended as the solution of the scheme (2.22)-(2.24) such that $\Psi_{J}=\Phi$ on $\omega_{M}^{\tau}$, with some $F$ such that $F_{j}^{m}=0$, for $j \geqslant J$ and $m \geqslant 1$, and $\Psi_{h}^{0}=0$. This completes the proof.

The corollary clarifies and proves condition (2.19) in the case of the discrete TBC; compare with the study of condition (2.9) for $E \equiv 1$ in Part I.

Let us go back to the scheme (2.5)-(2.8).

Proposition 2.7. Let the hypotheses of Proposition 2.3 be valid, with the following generalization of condition (2.19):

$$
\operatorname{Im}\left(\frac{1}{\hat{E}^{2}} \mathcal{S} \Phi, i \hbar \bar{\partial}_{t} \Phi+v \bar{s}_{t} \Phi\right)_{\omega_{M}^{\tau}} \geqslant 0 \text { for any } M \geqslant 1
$$

for some $\hat{E} \in N D\left(\bar{\omega}^{\tau}\right)$, any $\Phi$ such as in (2.19) and some $v \geqslant v_{0}$. Then the following bound holds:

$$
\max _{0 \leqslant m \leqslant M}\left\|\Psi^{m}\right\|_{\mathcal{H}_{h}+v I ; \bar{\omega}_{h}} \leqslant \hat{E}^{M}\left(\left\|\Psi_{h}^{0}\right\|_{\mathcal{H}_{h}+v I ; \bar{\omega}_{h}}+\frac{2}{\hbar} \sum_{m=1}^{M} \frac{1}{\hat{E}^{m}}\left\|F^{m}\right\|_{\mathcal{H}_{h}+v I ; \bar{\omega}_{h}} \tau_{m}\right),
$$

where $F^{m}$ is extended by $F_{0}^{m}=F_{J}^{m}=0$.

Proof. The argument is similar to that of Proposition 2.1. Namely, substituting $\Psi=\hat{E} Y$ into (2.11), dividing the result by $\hat{E}^{2}$ and using (2.18), we get the equality

$$
\frac{\hbar}{2}\left[\bar{\partial}_{t}\|Y\|_{\mathcal{H}_{h}+v I ; \bar{\omega}_{h}}^{2}+\frac{\bar{\partial}_{t}\left(\hat{E}^{2}\right)}{\hat{E}^{2}} \| \check{Y}_{\mathcal{H}_{h}+v I ; \bar{\omega}_{h}}^{2}\right]
$$




$$
=\operatorname{Im}\left\{\left(F, \frac{1}{\hat{E}^{2}}\left(\mathcal{H}_{h}+v I\right) \bar{s}_{t}(\hat{E} Y)\right)_{\omega_{h}}-\frac{\hbar^{2}}{2 \hat{E}^{2}} B_{1 \infty}\left(\mathcal{S} \Psi_{J}\right)\left(i \hbar \bar{\partial}_{t} \Psi_{J}+\bar{s}_{t} \Psi_{J}\right)^{*}\right\} .
$$

Since clearly

$$
\begin{aligned}
\left|\left(F, \frac{1}{\hat{E}^{2}}\left(\mathcal{H}_{h}+v I\right) \bar{s}_{t}(\hat{E} Y)\right)_{\omega_{h}}\right| & \leqslant\|F\|_{\mathcal{H}_{h}+v I ; \bar{\omega}_{h}} \frac{1}{\hat{E}^{2}}\left\|\bar{s}_{t}(\hat{E} Y)\right\|_{\mathcal{H}_{h}+v I ; \bar{\omega}_{h}} \\
& \leqslant\|F\|_{\mathcal{H}_{h}+v I ; \bar{\omega}_{h}} \frac{1}{\hat{E}} \bar{s}_{t}\|Y\|_{\mathcal{H}_{h}+v I ; \bar{\omega}_{h}},
\end{aligned}
$$

a standard argument leads to the following bound:

$$
\max _{0 \leqslant m \leqslant M}\left\|Y^{m}\right\|_{\mathcal{H}_{h}+v I ; \bar{\omega}_{h}} \leqslant\left\|Y^{0}\right\|_{\mathcal{H}_{h}+v I ; \bar{\omega}_{h}}+\frac{2}{\hbar} \sum_{m=1}^{M} \frac{1}{\hat{E}^{m}}\left\|F^{m}\right\|_{\mathcal{H}_{h}+v I ; \bar{\omega}_{h}} \tau_{m}
$$

for any $M \geqslant 1$. (Compare with (2.13).) The inverse substitution $Y=\Psi / \hat{E}$ yields the result.

REMARK 2.2. Taking into account identity (2.17), one can see that

$$
\|W\|_{\mathcal{H}_{h}+v I ; \bar{\omega}_{h}}^{2}=\mathcal{L}_{\bar{\omega}_{h}}(W, W)+v\|W\|_{\bar{\omega}_{h}}^{2} \geqslant 0
$$

(with $W_{0}=0$ ), and thus all the above results concerning the scheme (2.5)-(2.8) remain valid, for $v \geqslant v_{0}=-\lambda_{h \text { min }}$. Here $\lambda_{h \text { min }}$ is the minimal eigenvalue of the mesh selfadjoint real eigenvalue problem

$$
\mathcal{H}_{h} U=\lambda U \text { on } \omega_{h}, U_{0}=0, \quad \frac{\hbar^{2}}{2} B_{1 \infty} \bar{\partial}_{x} U_{J}+\frac{h}{2} V_{\infty} U_{J}=\frac{h}{2} \lambda U_{J} ;
$$

$\|\cdot\|_{\mathcal{H}_{h}+v I ; \bar{\omega}_{h}}$ is a semi-norm for $v=-\lambda_{h \min }$.

Corollary 2.8. Under conditions of any of Propositions 2.1, 2.3 and 2.7, the scheme (2.5)-(2.8) is uniquely solvable provided that $\mathcal{S}$ is a linear operator. $\mathcal{S}$

We give a result on the unique solvability of the scheme in the case of non-linear

Proposition 2.9. Let $\mathcal{S}^{m} \boldsymbol{\Phi}^{m}$ be affine in $\Phi^{m}$, that is, $\mathcal{S}^{m} \boldsymbol{\Phi}^{m}=\mathcal{S}_{0}^{m} \boldsymbol{\Phi}^{m-1}+$ $\left(\mathcal{S}_{1}^{m} \mathbf{\Phi}^{m-1}\right) \Phi^{m}$, and

$$
\hbar B_{1 \infty} \operatorname{Im} \mathcal{S}_{1}^{m} \mathbf{\Phi}^{m-1}>-\frac{h}{\tau_{m}}
$$

for any $\Phi$ such as in (2.9) and any $m \geqslant 1$. Then the scheme (2.5)-(2.8) is uniquely solvable.

Proof. Clearly, $\Psi^{m}$ satisfies the mesh problem

$$
\begin{gathered}
i \frac{\hbar}{\tau_{m}} \Psi^{m}-\frac{1}{2} \mathcal{H}_{h} \Psi^{m}=i \frac{\hbar}{\tau_{m}} \Psi^{m-1}+\frac{1}{2} \mathcal{H}_{h} \Psi^{m-1}+F^{m} \text { on } \omega_{h}, \\
\Psi_{0}^{m}=0,
\end{gathered}
$$




$$
\begin{array}{r}
{\left[\frac{\hbar^{2}}{4} B_{1 \infty} \bar{\partial}_{x} \Psi^{m}-\frac{h}{2}\left(i \frac{\hbar}{\tau_{m}}-\frac{1}{2} V_{\infty}\right) \Psi^{m}-\frac{\hbar^{2}}{2} B_{1 \infty}\left(\mathcal{S}_{1}^{m} \Psi_{J}^{m-1}\right) \Psi^{m}\right]_{J}} \\
=-\frac{\hbar^{2}}{4} B_{1 \infty} \bar{\partial}_{x} \Psi_{J}^{m-1}-\frac{h}{2}\left(i \frac{\hbar}{\tau_{m}}+\frac{1}{2} V_{\infty}\right) \Psi_{J}^{m-1}+\frac{\hbar^{2}}{2} B_{1 \infty} \mathcal{S}_{0}^{m} \Psi_{J}^{m-1}+\hat{G}^{m},
\end{array}
$$

which is a system of linear algebraic equations with a square matrix. Therefore it is sufficient to prove that the corresponding homogeneous system has only the trivial solution.

Taking the $(\cdot, \cdot)_{\omega_{h}}$-inner product of the homogeneous version of equation (2.32) and $\Psi^{m}$, applying the homogeneous version of boundary conditions (2.33) and identity (2.17) and then taking the imaginary part of the result, we obtain

$$
\frac{\hbar}{\tau_{m}}\left\|\Psi^{m}\right\|_{\omega_{h}}^{2}+\left(\frac{\hbar}{\tau_{m}} \frac{h}{2}+\frac{\hbar^{2}}{2} B_{1 \infty} \operatorname{Im}\left(\mathcal{S}_{1}^{m} \mathbf{\Psi}_{J}^{m-1}\right)\right)\left|\Psi_{J}^{m}\right|^{2}=0 ;
$$

compare with (2.11). Applying condition (2.31), we get $\Psi^{m}=0$, and the proof is complete.

Now we can prove stability bounds for the solution of the scheme (2.5)-(2.8) with respect to a perturbation in the operator $\mathcal{S}$. For any $\mathcal{E} \in N D\left(\bar{\omega}^{\tau}\right)$ and linear $\mathcal{S}$, we define the norm

$$
\|\mathcal{S}\|_{M, \mathcal{E}}:=\sup _{\Phi} \frac{\left\|\frac{1}{\mathcal{E}} \mathcal{S} \Phi\right\|_{\omega_{M}^{\tau}}}{\left\|\frac{1}{\mathcal{E}} \Phi\right\|_{\omega_{M}^{\tau}}},
$$

where sup is taken over all $\Phi: \bar{\omega}_{M}^{\tau} \rightarrow \mathbb{C}, \Phi^{0}=0$ and $\Phi \not \equiv 0$. Let $\mathcal{S}_{\text {ref }}$ be a linear operator satisfying condition (2.9) for $E \equiv 1$ and $\Psi_{\text {ref }}$ be the solution of the scheme for $\mathcal{S}=\mathcal{S}_{\text {ref }}$.

Proposition 2.10. Let $F=0, G=0$ and $\mathcal{S}$ be linear. For any $\mathcal{E} \in N D\left(\bar{\omega}^{\tau}\right)$ and $M \geqslant 1$, the following claims are valid.

1. If condition (2.9) holds, then

$$
\max _{0 \leqslant m \leqslant M}\left\|\Psi_{\mathrm{ref}}^{m}-\Psi^{m}\right\|_{\bar{\omega}_{h}} \leqslant \frac{2}{h} \hbar B_{1 \infty}\|\mathcal{E}\|_{\omega_{M}^{\tau}}\left\|\frac{E}{\mathcal{E}}\right\|_{\omega_{M}^{\tau}}\left\|\Psi_{h}^{0}\right\|_{\bar{\omega}_{h}}\left\|\mathcal{S}_{\mathrm{ref}}-\mathcal{S}\right\|_{M, \mathcal{E}}
$$

2. If condition (2.30) holds, then

$$
\begin{aligned}
& \max _{0 \leqslant m \leqslant M}\left\|\Psi_{\text {ref }}^{m}-\Psi^{m}\right\|_{\bar{\omega}_{h}} \\
& \leqslant \sqrt{\frac{2 X}{\nu_{1} h}} \hbar B_{1 \infty}\|\mathcal{E}\|_{\omega_{M}^{\tau}}\left\|\frac{\hat{E}}{\mathcal{E}}\right\|_{\omega_{M}^{\tau}}\left\|\Psi_{h}^{0}\right\|_{\mathcal{H}_{h}+v I ; \bar{\omega}_{h}}\left\|\mathcal{S}_{\text {ref }}-\mathcal{S}\right\|_{M, \mathcal{E}} .
\end{aligned}
$$

3. If both condition (2.19) for $\mathcal{S}=\mathcal{S}_{\text {ref }}$ and condition (2.30) hold, then

$$
\leqslant \frac{1}{\sqrt{\nu_{1}}}\left(\sqrt{2 X} \hbar B_{1 \infty}\|\sqrt{\hat{E}+1} \mathcal{E}\|_{\omega_{M}^{\tau}}\left\|\frac{\hat{E}}{\mathcal{E}}\right\|_{\omega_{M}^{\tau}}\right)^{2 / 3}\left\|\Psi_{\mathrm{ref}}^{m}-\Psi_{h}^{0}\right\|_{\mathcal{H}_{h}+v I ; \bar{\omega}_{h}}\left\|\mathcal{S}_{\mathrm{ref}}-\mathcal{S}\right\|_{M, \mathcal{E}}^{2 / 3} .
$$


REMARK 2.3. In Claims 1 and 2, the bounds are proportional to $\left\|\mathcal{S}_{\text {ref }}-\mathcal{S}\right\|_{M, \mathcal{E}}$ but they contain multipliers proportional to $1 / h$ and $1 / \sqrt{h}$. In Claim 3 , the bound is proportional only to $\left\|\mathcal{S}_{\text {ref }}-\mathcal{S}\right\|_{M, \mathcal{E}}^{2 / 3}$, but this is uniform in $h$.

Proof. Clearly, the difference $\Delta \Psi:=\Psi_{\text {ref }}-\Psi$ satisfies the scheme

$$
\begin{aligned}
i \hbar \bar{\partial}_{t} \Delta \Psi & =\mathcal{H}_{h} \bar{s}_{t} \Delta \Psi \text { on } \omega_{h} \times \omega^{\tau} \\
\Delta \Psi_{0}^{m} & =0 \text { for } m \geqslant 1 \\
\mathcal{D}_{\Gamma} \Delta \Psi_{J}^{m} & =\frac{\hbar^{2}}{2} B_{1 \infty} \mathcal{S}_{\mathrm{ref}}^{m} \Delta \boldsymbol{\Psi}_{J}^{m}+\frac{\hbar^{2}}{2} B_{1 \infty}\left(\mathcal{S}_{\mathrm{ref}}^{m}-\mathcal{S}^{m}\right) \boldsymbol{\Psi}_{J}^{m} \text { for } m \geqslant 1, \\
\Delta \Psi^{0} & =0 \text { on } \bar{\omega}_{h} .
\end{aligned}
$$

Using Proposition 2.1 for $\Delta \Psi$ (replacing $\Psi$ ), $\mathcal{S}=\mathcal{S}_{\text {ref }}$ and $E \equiv 1$, we get

$$
\begin{aligned}
& \max _{0 \leqslant m \leqslant M}\left\|\Psi_{\text {ref }}^{m}-\Psi^{m}\right\|_{\bar{\omega}_{h}} \\
\leqslant & \sqrt{\frac{2}{h}} \hbar B_{1 \infty} \sum_{m=1}^{M}\left|\left(\mathcal{S}_{\text {ref }}^{m}-\mathcal{S}^{m}\right) \Psi_{J}^{m}\right| \tau_{m} \\
\leqslant & \sqrt{\frac{2}{h}} \hbar B_{1 \infty}\|\mathcal{E}\|_{\omega_{M}^{\tau}}\left\|\frac{1}{\mathcal{E}}\left(\mathcal{S}_{\text {ref }}-\mathcal{S}\right) \Psi_{J}\right\|_{\omega_{M}^{\tau}} \\
\leqslant & \sqrt{\frac{2}{h}} \hbar B_{1 \infty}\|\mathcal{E}\|_{\omega_{M}^{\tau}}\left\|\mathcal{S}_{\text {ref }}-\mathcal{S}\right\|_{M, \mathcal{E}}\left\|\frac{E}{\mathcal{E}}\right\|_{\omega_{M}^{\tau}} \max _{1 \leqslant m \leqslant M} \frac{1}{E^{m}}\left|\Psi_{J}^{m}\right|
\end{aligned}
$$

Exploiting the estimate $\left|W_{J}\right| \leqslant \sqrt{2 / h}\|W\|_{\bar{\omega}_{h}}$ and Proposition 2.1, we establish Claim 1.

Replacing $\hat{E}$ by $E$ in (2.35) and using another estimate $\left|W_{J}\right| \leqslant$ $\sqrt{X / \nu_{1}}\|W\|_{\mathcal{H}_{h}+v I ; \bar{\omega}_{h}}$ (with $W_{0}=0$ ) and Proposition 2.7, we prove Claim 2 as well.

To establish Claim 3, we apply Corollary 2.2 instead of Proposition 2.1 together with Proposition 2.3 (for $\mathcal{S}=\mathcal{S}_{\text {ref }}$ ) and Proposition 2.7 and obtain

$$
\begin{gathered}
\max _{0 \leqslant m \leqslant M}\left\|\Psi_{\text {ref }}^{m}-\Psi^{m}\right\|_{\bar{\omega}_{h}} \\
\leqslant\left[\hbar B_{1 \infty}\left(\frac{2}{\sqrt{\nu_{1}}}\left\|\Psi_{h}^{0}\right\|_{\mathcal{H}_{h}+v I ; \bar{\omega}_{h}}\right)^{1 / 2}\|\sqrt{\hat{E}+1} \mathcal{E}\|_{\omega_{M}^{\tau}}\left\|\frac{1}{\mathcal{E}}\left(\mathcal{S}_{\text {ref }}-\mathcal{S}\right) \Psi_{J}\right\|_{\omega_{M}^{\tau}}\right]^{2 / 3} .
\end{gathered}
$$

An argument similar to that just used to prove Claim 2 completes the proof.

Note that, for linear $\mathcal{S}_{\text {ref }}$, the above equations for $\Psi_{\text {ref }}^{m}-\Psi^{m}$ are linear, and one can generalize all the bounds for a nonlinear $\mathcal{S}$.

REMARK 2.4. The proposition may be modified as follows. Assume that condition (2.9) is valid in Claims 1-3 and that condition (2.19) for $\mathcal{S}=\mathcal{S}_{\text {ref }}$ replaces condition (2.30) in Claim 2 (Probably the last moment is the most interesting.) Then similar bounds for $\Psi_{\text {ref }}-\Psi$ hold, provided that the multiplier $E^{M}\left\|\frac{\mathcal{E}}{E}\right\|_{\omega_{M}^{\tau}}\left\|\frac{1}{\mathcal{E}}\right\|_{\omega_{M}^{\tau}}$ replaces $\|\mathcal{E}\|_{\omega_{M}^{\tau}}\left\|\frac{E}{\mathcal{E}}\right\|_{\omega_{M}^{\tau}}$ and $\|\mathcal{E}\|_{\omega_{M}^{\tau}}\left\|\frac{\hat{E}}{\mathcal{E}}\right\|_{\omega_{M}^{\tau}}$ in Claims 1 and 2 and that the multiplier $E^{M}\left\|\sqrt{\hat{E}+1} \frac{\mathcal{E}}{E}\right\|_{\omega_{M}^{\tau}}\left\|\frac{1}{\mathcal{E}}\right\|_{\omega_{M}^{\tau}}$ replaces $\|\sqrt{\hat{E}+1} \mathcal{E}\|_{\omega_{M}^{\tau}}\left\|\frac{\hat{E}}{\mathcal{E}}\right\|_{\omega_{M}^{\tau}}$ in Claim 3. 
To check this, one just rewrites the boundary condition (2.34) in the form

$$
\mathcal{D}_{\Gamma} \Delta \Psi_{J}^{m}=\frac{\hbar^{2}}{2} B_{1 \infty} \mathcal{S}^{m} \Delta \Psi_{J}^{m}+\frac{\hbar^{2}}{2} B_{1 \infty}\left(\mathcal{S}_{\text {ref }}^{m}-\mathcal{S}^{m}\right) \boldsymbol{\Psi}_{\text {ref } J}^{m} \text { for } m \geqslant 1,
$$

and applies Proposition 2.1 in the original form (not for $\mathcal{S}=\mathcal{S}_{\text {ref }}$ and $E \equiv 1$ as above) to derive bounds for $\Delta \Psi$. On the other hand, bounds (2.10) with $E \equiv 1$ and $(2.20)$ for $\Psi=\Psi_{\text {ref }}$ replace the previous corresponding bounds for $\Psi$.

REMARK 2.5. The multiplicative inequality (2.16) leads to uniform bounds provided that the assumptions of Claim 3 are valid. In particular, Claim 3 itself implies that

$$
\begin{gathered}
\max _{0 \leqslant m \leqslant M}\left\|\Psi_{\mathrm{ref}}^{m}-\Psi^{m}\right\|_{C\left(\bar{\omega}_{h}\right)} \\
\leqslant \sqrt{2}\left(\max _{0 \leqslant m \leqslant M}\left\|\bar{\partial}_{x} \Psi_{\mathrm{ref}}^{m}\right\|_{\widetilde{\omega}_{h}}+\max _{0 \leqslant m \leqslant M}\left\|\bar{\partial}_{x} \Psi^{m}\right\|_{\widetilde{\omega}_{h}}\right)^{1 / 2} \cdot \max _{0 \leqslant m \leqslant M}\left\|\Psi_{\mathrm{ref}}^{m}-\Psi^{m}\right\|_{\bar{\omega}_{h}}^{1 / 2} \\
\leqslant \sqrt{\frac{2}{\nu_{1}}}\left(\sqrt{2 X} \hbar B_{1 \infty}\right)^{1 / 3} \sqrt{\hat{E}^{M}+1}\|\sqrt{\hat{E}+1} \mathcal{E}\|_{\omega_{M}^{\tau}}^{1 / 3}\left\|\frac{\hat{E}}{\mathcal{E}}\right\|_{\omega_{M}^{\tau}}^{1 / 3} \\
\cdot\left\|\Psi_{h}^{0}\right\|_{\mathcal{H}_{h}+v I ; \bar{\omega}_{h}}\left\|\mathcal{S}_{\mathrm{ref}}-\mathcal{S}\right\|_{M, \mathcal{E}}^{1 / 3} .
\end{gathered}
$$

REMARK 2.6. It is not difficult to generalize Proposition 2.10 (and the previous remarks) as follows. Let

$$
\|Y\|_{L^{p}\left(\omega_{M}^{\tau}\right)}:=\left(\sum_{m=1}^{M}\left|Y^{m}\right|^{p} \tau_{m}\right)^{1 / p} \quad \text { for } 1 \leqslant p<\infty
$$

and

$$
\|Y\|_{L^{p}\left(\omega_{M}^{\tau}\right)}:=\max _{1 \leqslant m \leqslant M}\left|Y^{m}\right| \text { for } p=\infty .
$$

Let $p^{\prime}$ satisfy $1 / p^{\prime}+1 / p=1$.

Given $p, p_{1} \in[1, \infty]$, we define the more general operator norm

$$
\|\mathcal{S}\|_{M, \mathcal{E}, p, p_{1}}:=\sup _{\Phi} \frac{\left\|\frac{1}{\mathcal{E}} \mathcal{S} \Phi\right\|_{L^{p_{1}\left(\omega_{M}^{\tau}\right)}}}{\left\|\frac{1}{\mathcal{E}} \Phi\right\|_{L^{p}\left(\omega_{M}^{\tau}\right)}}
$$

clearly, $\|\mathcal{S}\|_{M, \mathcal{E}}=\|\mathcal{S}\|_{M, \mathcal{E}, p, p_{1}}$ for $p=p_{1}=2$. Then one can replace, in the bounds of Proposition 2.10 , the norm $\|\cdot\|_{\omega_{M}^{\tau}}$ respectively by $\|\cdot\|_{L^{p_{1}^{\prime}\left(\omega_{M}^{\tau}\right)}}$ for $\mathcal{E}$ and $\sqrt{\hat{E}+1} \mathcal{E}$ and by $\|\cdot\|_{L^{p}\left(\omega_{M}^{\tau}\right)}$ for $\frac{E}{\mathcal{E}}$ and $\frac{\hat{E}}{\mathcal{E}}$, together with $\left\|\mathcal{S}_{\text {ref }}-\mathcal{S}\right\|_{M, \mathcal{E}}$ by $\left\|\mathcal{S}_{\text {ref }}-\mathcal{S}\right\|_{M, \mathcal{E}, p, p_{1}}$.

Indeed, for example, to generalize Claim 1, one can write

$$
\begin{aligned}
\left\|\left(\mathcal{S}_{\mathrm{ref}}-\mathcal{S}\right) \boldsymbol{\Phi}\right\|_{L^{1}\left(\omega_{M}^{\tau}\right)} & \leqslant\|\mathcal{E}\|_{L^{p_{1}^{\prime}\left(\omega_{M}^{\tau}\right)}}\left\|\frac{1}{\mathcal{E}}\left(\mathcal{S}_{\mathrm{ref}}-\mathcal{S}\right) \boldsymbol{\Phi}\right\|_{L^{p_{1}\left(\omega_{M}^{\tau}\right)}} \\
& \leqslant\|\mathcal{E}\|_{L^{p_{1}^{\prime}\left(\omega_{M}^{\tau}\right)}}\left\|\mathcal{S}_{\mathrm{ref}}-\mathcal{S}\right\|_{M, \mathcal{E}, p, p_{1}}\left\|\frac{1}{\mathcal{E}} \Phi\right\|_{L^{p}\left(\omega_{M}^{\tau}\right)} \\
& \leqslant\|\mathcal{E}\|_{L^{p_{1}^{\prime}\left(\omega_{M}^{\tau}\right)}}\left\|\frac{E}{\mathcal{E}}\right\|_{L^{p}\left(\omega_{M}^{\tau}\right)}\left\|\frac{1}{E} \Phi\right\|_{L^{\infty}\left(\omega_{M}^{\tau}\right)}\left\|\mathcal{S}_{\mathrm{ref}}-\mathcal{S}\right\|_{M, \mathcal{E}, p, p_{1}} .
\end{aligned}
$$




\section{The case of discrete convolution-type operators $\mathcal{S}$}

Let the mesh $\bar{\omega}^{\tau}$ be uniform (that is, $\tau_{m}=\tau$ for $m \geqslant 1$ ) in this section. We assume now that $\mathcal{S}$ is a discrete convolution operator of the form

$$
\mathcal{S} \boldsymbol{\Phi}=Q * \bar{s}_{t} \Phi,
$$

for some kernel $Q: \bar{\omega}^{\tau} \rightarrow \mathbb{C}$ and any $\Phi: \bar{\omega}^{\tau} \rightarrow \mathbb{C}, \Phi^{0}=0$. Hereafter $\left.\Phi^{-1} \equiv \Phi^{m}\right|_{m=-1}:=0$ and

$$
(Q * \Phi)^{m}:=\sum_{n=0}^{m} Q^{n} \Phi^{m-n} \text { for } m \geqslant 0 .
$$

We apply the technique of reproducing functions and define, for any $\Phi: \bar{\omega}^{\tau} \rightarrow \mathbb{C}$, the power series

$$
\mathcal{T}[\Phi](z):=\sum_{m=0}^{\infty} \Phi^{m} z^{m} .
$$

If $\Phi$ satisfies the bound $\left|\Phi^{m}\right| \leqslant C \rho^{m}$ for any $m \geqslant 0$ and some $\rho>0$, then $\mathcal{T}[\Phi]$ is analytic in the disc $D_{1 / \rho}:=\{z \in \mathbb{C}:|z|<1 / \rho\}$. We set $q:=\mathcal{T}[Q]$.

We recall that the operator of the discrete TBC, see Corollary 2.6, may be written in the form, see [12] and Part I:

$$
\mathcal{S}_{\text {ref }} \boldsymbol{\Phi}=Q_{\text {ref }} * \bar{s}_{t} \Phi
$$

where the kernel $Q_{\text {ref }}$ is such that

$$
\begin{gathered}
q_{\mathrm{ref}}(z):=\mathcal{T}\left[Q_{\mathrm{ref}}\right](z)=\frac{1}{h} \sqrt{\gamma^{2}(z)-1}, \\
\gamma(z):=1+a_{0}-i a_{1} \frac{1-z}{1+z}, \quad a_{0}:=\frac{h^{2} V_{\infty}}{\hbar^{2} B_{1 \infty}}, \quad a_{1}:=\frac{2 h^{2}}{\tau \hbar B_{1 \infty}} ;
\end{gathered}
$$

$\sqrt{w}$ denotes the analytic branch of $\sqrt{w}$ in $\mathbb{C}$ with the cross-cut along the positive real semi-axis $\operatorname{Re} w>0, \operatorname{Im} w=0$, such that $\sqrt{-1}=i$.

Let $H^{s}\left(D_{r}\right), 1 \leqslant s<\infty$, be the Hardy space of functions $p$ analytic in $D_{r}$ such that

$$
\sup _{0<\rho<r} \int_{0}^{2 \pi}\left|p\left(\rho e^{i \varphi}\right)\right|^{s} d \varphi<\infty .
$$

For such functions, the trace $\left.p\right|_{\partial D_{r}} \in L^{s}\left(\partial D_{r}\right)$ is well defined (for example see [18]).

Proposition 3.1. Let $0<r \leqslant 1$.

1. The operator $\mathcal{S}$ satisfies condition (2.9) for $E^{m} \equiv r^{-m}$ if and only if $q$ is analytic in $D_{r}$ and

$$
\operatorname{Im} q(z) \geqslant 0 \text { on } D_{r} .
$$

2. Let $q$ be analytic in $D_{r}$. The operator $\mathcal{S}$ satisfies condition (2.30) for $\hat{E}^{m} \equiv$ $r^{-m}$ and some real $v$ if and only if

$$
\operatorname{Im}\left\{q(z)\left[\frac{\hbar^{2} B_{1 \infty}}{h^{2}}(1-\gamma(z))^{*}+v+V_{\infty}\right]\right\} \geqslant 0 \text { on } D_{r} .
$$


REMARK 3.1. Let $p$ be analytic in $D_{r}$. The condition

$$
\operatorname{Im} p(z) \geqslant 0 \text { on } D_{r}
$$

is equivalent to $\operatorname{Im} p(z)>0$ on $D_{r}$ provided that $p(z) \not \equiv 0$ on $D_{r}$. Moreover, condition (3.4) is equivalent to $\operatorname{Im} p(z) \geqslant 0$ almost everywhere on $\partial D_{r}$ provided that $p \in H^{1}\left(D_{r}\right)$ (in particular, $p \in C\left(\bar{D}_{r}\right)$ ). These properties are implied by the maximum principle for harmonic functions (since $\operatorname{Im} p$ is harmonic in $D_{r}$ ).

Proof.

1. Actually, Claim 1 follows from [14], Chapter 9. We give a somewhat different proof both in order to develop a technique allowing to derive other results in the section and for completeness.

We first fix $M \geqslant 1$ and values $\Phi^{1}, \Phi^{2}, \ldots, \Phi^{M}$ together with $\Phi^{-1}=\Phi^{0}=0$. In order to satisfy $\bar{s}_{t} \Phi^{m}=0$ for $m>M$, we set $\Phi^{m}=(-1)^{m-M} \Phi^{M}$ for $m>M$ and we get, for $E^{m} \equiv \rho^{-m}$ with $0<\rho \leqslant r$

$$
\begin{aligned}
S_{1}(\rho):=\left(\frac{1}{E^{2}} \mathcal{S} \boldsymbol{\Phi}, \bar{s}_{t} \Phi\right)_{\omega_{M}^{\tau}} & =\tau \sum_{m=0}^{\infty} \frac{1}{\left(E^{m}\right)^{2}} \mathcal{S}^{m} \boldsymbol{\Phi}^{m}\left(\bar{s}_{t} \Phi^{m}\right)^{*} \\
& =\tau \sum_{m=0}^{\infty}\left(\frac{Q}{E} * \frac{\Lambda}{E}\right)^{m} \frac{\left(\Lambda^{m}\right)^{*}}{E^{m}}
\end{aligned}
$$

where $\Lambda:=\bar{s}_{t} \Phi$. Note that $\mathcal{T}[\Lambda]$ is a polynomial with degree not greater than $M$.

For $q$ analytic in $D_{r}$, we apply Parseval's equality for the complex trigonometric series and the formulas

$$
\mathcal{T}[Q * \Lambda]=\mathcal{T}[Q] * \mathcal{T}[\Lambda], \quad \mathcal{T}\left[\left\{\rho^{m} \Lambda^{m}\right\}_{m=0}^{\infty}\right](z)=\mathcal{T}[\Lambda](\rho z),
$$

and we obtain, for $0<\rho<r$

$$
\begin{aligned}
S_{1}(\rho) & =\frac{\tau}{2 \pi} \int_{0}^{2 \pi} \mathcal{T}\left[\frac{Q}{E} * \frac{\Lambda}{E}\right]\left(e^{i \varphi}\right)\left(\mathcal{T}\left[\frac{\Lambda}{E}\right]\left(e^{i \varphi}\right)\right)^{*} d \varphi \\
& =\frac{\tau}{2 \pi} \int_{0}^{2 \pi} q\left(\rho e^{i \varphi}\right)\left|\mathcal{T}[\Lambda]\left(\rho e^{i \varphi}\right)\right|^{2} d \varphi .
\end{aligned}
$$

Therefore

$$
\operatorname{Im} S_{1}(\rho)=\frac{\tau}{2 \pi} \int_{0}^{2 \pi} \operatorname{Im} q\left(\rho e^{i \varphi}\right)\left|\mathcal{T}[\Lambda]\left(\rho e^{i \varphi}\right)\right|^{2} d \varphi .
$$

Inequality (3.2) implies that $\operatorname{Im} S_{1}(\rho) \geqslant 0$, and since $S_{1}(r)=\lim _{\rho \rightarrow r_{-}} S_{1}(\rho)$, we get that $\operatorname{Im} S_{1}(r) \geqslant 0$ too.

Conversely, let $\operatorname{Im} S_{1}(r) \geqslant 0$. Firstly, taking $\Lambda^{n}=0$ for $n \neq 1, m(2 \leqslant m \leqslant$ $M)$, we get

$$
S_{1}(r)=Q^{0}\left|r \Lambda^{1}\right|^{2}+r^{m-1} Q^{m-1} r \Lambda^{1}\left(r^{m} \Lambda^{m}\right)^{*}+Q^{0}\left|r^{m} \Lambda^{m}\right|^{2}
$$

and consequently

$$
\operatorname{Im} S_{1}(r)=\operatorname{Im} Q^{0}\left(\left|r \Lambda^{1}\right|^{2}+\left|r^{m} \Lambda^{m}\right|^{2}\right)
$$




$$
+\frac{1}{2 i}\left(r^{m-1} Q^{m-1} r \Lambda^{1}\left(r^{m} \Lambda^{m}\right)^{*}-\left(r^{m-1} Q^{m-1}\right)^{*}\left(r \Lambda^{1}\right)^{*} r^{m} \Lambda^{m}\right) .
$$

This Hermitian quadratic form is non-negative if and only if $r^{m-1}\left|Q^{m-1}\right| \leqslant$ $2 \operatorname{Im} Q^{0}$; the bound implies analyticity of $q$ in $D_{r}$.

Secondly, we show that

$$
\operatorname{Im} S_{1}(\rho) \geqslant 0 \text { for } 0<\rho<r .
$$

To this end, we set

$$
\lambda_{1}(\rho):=\min _{\|\Lambda\|_{\omega_{M}^{\tau}}^{\tau}=1} \operatorname{Im} S_{1}(\rho) \text { for } 0<\rho<r
$$

Let the point of minimum be $\Lambda_{0}$. We set $A:=\Lambda_{0} / E$ and $A^{m}(\varphi):=e^{-i m \varphi} A^{m}$ and get

$$
\begin{aligned}
\lambda_{1}(\rho) & =\operatorname{Im}\left(\frac{Q}{E} * A, A\right)_{\omega_{M}^{\tau}} \\
& \geqslant \min _{0 \leqslant \varphi \leqslant 2 \pi} \operatorname{Im}\left(\frac{Q}{E} * A(\varphi), A(\varphi)\right)_{\omega_{M}^{\tau}}=\min _{0 \leqslant \varphi \leqslant 2 \pi} \operatorname{Im} F\left(\rho e^{i \varphi}\right),
\end{aligned}
$$

where once again $E^{m} \equiv \rho^{-m}$ and

$$
F(z):=\left.((Z Q) * A, A)_{\omega_{M}^{\tau}}\right|_{Z^{m}=z^{m}}
$$

is a polynomial. By the maximum principle applied to the harmonic function $\operatorname{Im} F$, we obtain $\min _{|z|=\rho} \operatorname{Im} F(z) \geqslant \min _{|z|=r} \operatorname{Im} F(z)$. Since

$$
F\left(r e^{i \varphi}\right)=\left.\operatorname{Im} S_{1}(r)\right|_{\Lambda^{m} \equiv r^{-m} A^{m}(\varphi)} \geqslant 0,
$$

we get $\lambda_{1}(\rho) \geqslant 0$, and thus inequality (3.8) is valid.

Finally, $z^{-1} \mathcal{T}[\Lambda](z)$ is an arbitrary polynomial with degree not greater than $M-1$. Consequently, for $M=2(N+1)$, the expression

$$
\left.\left\{z^{-1} \mathcal{T}[\Lambda](z)\right\}\right|_{z=r e^{i \varphi}} e^{-i N \varphi}=\sum_{k=-N}^{N} c_{k} e^{i k \varphi}=: T_{N}(\varphi),
$$

is an arbitrary complex trigonometric polynomial with degree not greater than $N$. By virtue of (3.7) and (3.8) we get

$$
\int_{0}^{2 \pi} \operatorname{Im} q\left(\rho e^{i \varphi}\right)|g(\varphi)|^{2} d \varphi \geqslant 0 \text { for any } g=T_{N} .
$$

Since the set of trigonometric polynomials is dense in $L^{2}(0,2 \pi)$, inequality (3.10) is valid for any $g \in L^{2}(0,2 \pi)$ as well, taking into account the inequality, for any $\varkappa \in L^{\infty}(0,2 \pi), f, g \in L^{2}(0,2 \pi)$

$$
\left.\left|\int_{0}^{2 \pi} \varkappa\right| f\right|^{2} d \varphi-\int_{0}^{2 \pi} \varkappa|g|^{2} d \varphi \mid \leqslant\|\varkappa\|_{L^{\infty}(0,2 \pi)}\|f-g\|_{L^{2}(0,2 \pi)}\|f+g\|_{L^{2}(0,2 \pi)} .
$$

Consequently, $\operatorname{Im} q(z) \geqslant 0$ on $\partial D_{\rho}$ for any $0<\rho<r$, and inequality (3.2) is proved. 
2. Once again we first fix $M \geqslant 1$ and values $\Phi^{1}, \Phi^{2}, \ldots, \Phi^{M}$ together with $\Phi^{-1}=$ $\Phi^{0}=0$. To satisfy $\left(i \hbar \bar{\partial}_{t} \Phi+v \bar{s}_{t} \Phi\right)^{m}=0$ for $m>M$, we set

$$
\Phi^{m}:=\beta^{m-M} \Phi^{M} \text { for } m>M \text { with } \beta:=\frac{1+i \tau v /(2 \hbar)}{1-i \tau v /(2 \hbar)} .
$$

Similarly to formulas (3.5) we get, for $\hat{E}^{m}=\rho^{-m}$ with $0<\rho \leqslant r$

$$
\begin{aligned}
S_{2}(\rho): & =\left(\frac{1}{\hat{E}^{2}} \mathcal{S} \Phi, i \hbar \bar{\partial}_{t} \Phi+v \bar{s}_{t} \Phi\right)_{\omega_{M}^{\tau}} \\
& =\tau \sum_{m=0}^{\infty}\left(\frac{Q}{\hat{E}} * \frac{\bar{s}_{t} \Phi}{\hat{E}}\right)^{m} \frac{\left(i \hbar \bar{\partial}_{t} \Phi^{m}+v \bar{s}_{t} \Phi^{m}\right)^{*}}{\hat{E}^{m}}
\end{aligned}
$$

Since $|\beta|=1$, we have $\sup _{m \geqslant 0}\left|\Phi^{m}\right|=\max _{1 \leqslant m \leqslant M}\left|\Phi^{m}\right|$, and therefore $\mathcal{T}[\Phi](z)$ and $\mathcal{T}\left[\bar{s}_{t} \Phi\right](z)=((1+z) / 2) \mathcal{T}[\Phi](z)$ are analytic in $D_{1}$. Moreover, for $z \in D_{1}$

$$
\mathcal{T}\left[i \hbar \bar{\partial}_{t} \Phi\right](z)=i \frac{2 \hbar}{\tau} \frac{1-z}{1+z} \mathcal{T}\left[\bar{s}_{t} \Phi\right](z)=\left[\frac{\hbar^{2} B_{1 \infty}}{h^{2}}(1-\gamma(z))+V_{\infty}\right] \mathcal{T}\left[\bar{s}_{t} \Phi\right](z)
$$

For $q$ analytic in $D_{r}$ and $0<\rho<r$, similarly to (3.6) we obtain

$$
\begin{gathered}
S_{2}(\rho)=\frac{\tau}{2 \pi} \int_{0}^{2 \pi}\left\{q(z)\left[\frac{\hbar^{2} B_{1 \infty}}{h^{2}}(1-\gamma(z))+\left(v+V_{\infty}\right)\right]^{*}\right. \\
\left.\cdot\left|\mathcal{T}\left[\bar{s}_{t} \Phi\right](z)\right|^{2}\right\}\left.\right|_{z=\rho e^{i \varphi}} d \varphi
\end{gathered}
$$

and thus, after setting $p(z):=q(z)\left[\left(\hbar^{2} B_{1 \infty} / h^{2}\right)(1-\gamma(z))^{*}+\left(v+V_{\infty}\right)\right]$,

$$
\operatorname{Im} S_{2}(\rho)=\frac{\tau}{2 \pi} \int_{0}^{2 \pi} \operatorname{Im} p\left(\rho e^{i \varphi}\right)\left|\mathcal{T}\left[\bar{s}_{t} \Phi\right]\left(\rho e^{i \varphi}\right)\right|^{2} d \varphi
$$

Inequality (3.3) implies that $\operatorname{Im} S_{2}(\rho) \geqslant 0$, and since $S_{2}(r)=\lim _{\rho \rightarrow r_{-}} S_{2}(\rho)$, we get that $\operatorname{Im} S_{2}(r) \geqslant 0$ too.

Conversely, let $\operatorname{Im} S_{2}(r) \geqslant 0$. Comparing (3.6) and (3.11), in the case $\Phi^{M}=0$, we get another representation for $S_{2}(\rho)$ :

$$
S_{2}(\rho)=\left(\frac{P}{E} * \frac{\Lambda}{E}, \frac{\Lambda}{E}\right)_{\omega_{M}^{\tau}},
$$

where $P$ is such that $\mathcal{T}[P]=p, E^{m} \equiv \rho^{-m}$ and $\Lambda=\bar{s}_{t} \Phi$. Consequently by analogy with (3.8) we have (provided that $\Phi^{M}=0$ )

$$
\operatorname{Im} S_{2}(\rho) \geqslant 0 \text { for } 0<\rho<r
$$

Furthermore, for $\Phi^{M}=0$, we find that

$$
z^{-1} \mathcal{T}\left[\bar{s}_{t} \Phi\right](z)=\sum_{m=0}^{M-2} \Theta_{m} z^{m}
$$


is any polynomial of degree not greater than $M-2$ such that $\sum_{m=0}^{M-2}(-1)^{m} \Theta_{m}=0$. Therefore, for $M=2 N+3$, the expression (3.9) is an arbitrary trigonometric polynomial $T_{N}$ such that $T_{N}(\pi)=0$. By virtue of (3.12) and (3.14) we get

$$
\int_{0}^{2 \pi} \operatorname{Im} p\left(\rho e^{i \varphi}\right)|g(\varphi)|^{2} d \varphi \geqslant 0
$$

for any $g=T_{N}$. With the help of the above density argument, the inequality holds also for any $g \in L^{2}(0,2 \pi)$. Consequently, $\operatorname{Im} p(z) \geqslant 0$ on $\partial D_{\rho}$ for any $0<\rho<r$, and inequality (3.3) is proved.

Note that the regularizing role of the multipliers $1 / E^{2}$ and $1 / \hat{E}^{2}$ in conditions (2.9) and (2.30) is clear from the statement and the proof of Proposition 3.1; recall also that the most interesting choice of $r$ is $r(\tau)=1 /\left(1+c_{0} \tau\right)$ with $c_{0} \geqslant 0$.

Let us apply the last proposition for $q=q_{\text {ref }}$.

Proposition 3.2. For $\mathcal{S}=\mathcal{S}_{\text {ref }}$, inequalities (2.9) and (2.30) hold for $E^{m}=\hat{E}^{m} \equiv$ $r^{-m}$ with any $0<r \leqslant 1$ and for any $v \geqslant-V_{\infty}$.

Proof. Recall that $q_{\text {ref }}$ is analytic, with $\operatorname{Im} q_{\text {ref }}(z)>0$, in $D_{1}$ (see Part I). Consequently according to Proposition 3.1, it is sufficient to check the inequality

$$
\operatorname{Im}\left[q_{\text {ref }}(z)(1-\gamma(z))^{*}\right]>0 \text { in } D_{1},
$$

which implies (3.3) for $q=q_{\mathrm{ref}}, r=1$ and $v \geqslant-V_{\infty}$.

Since the linear-fractional function $z \rightarrow \zeta=\gamma(z)$ establishes a one-to-one correspondence between $D_{1}$ and the lower half-plane $\{\operatorname{Im} \zeta<0\}$, inequality (3.15) can be rewritten in the form

$$
\operatorname{Im}\left(\sqrt{\zeta^{2}-1}\left(1-\zeta^{*}\right)\right)>0 \text { for } \operatorname{Im} \zeta<0 .
$$

We transform this as follows

$$
\operatorname{Im}\left(\sqrt[-]{w(w+2)} w^{*}\right)<0 \text { for } \operatorname{Im} w<0
$$

and set $w=t e^{i \theta}-1$ with $t>0$ and $\pi<\theta<2 \pi$. Since $\arg \left(t e^{i \theta}+2\right)$ runs over $(\theta, 2 \pi)$, we get

$$
\begin{aligned}
\sin \arg \left(\sqrt{w(w+2)} w^{*}\right) & =\sin \left(\frac{1}{2}\left(\theta+\arg \left(t e^{i \theta}+2\right)\right)-\pi-\theta\right) \\
& =-\sin \frac{\arg \left(t e^{i \theta}+2\right)-\theta}{2}<0
\end{aligned}
$$

which proves the required inequality.

Note that, for $\mathcal{S}=\mathcal{S}_{\text {ref }}$ and $E \equiv 1$, two other proofs of inequality (2.9) were given in Part I.

Now we turn to estimates for $\mathcal{S}_{\text {ref }}-\mathcal{S}$. In contrast to [5], to this end we find it essential to rewrite (3.1) in the equivalent form

$$
\mathcal{S} \Phi=R * \Phi \text { with } R:=\bar{s}_{t} Q,
$$


where we set $Q^{-1}=0$. Clearly, $\mathcal{T}[R](z)=((1+z) / 2) \mathcal{T}[Q](z)$ and, in particular, $\mathcal{T}\left[R_{\mathrm{ref}}\right](z)=((1+z) / 2) q_{\mathrm{ref}}(z)$, for $R_{\mathrm{ref}}:=\bar{s}_{t} Q_{\mathrm{ref}}$ (with $\left.Q_{\mathrm{ref}}^{-1}=0\right)$; note that an explicit expression for $R_{\text {ref }}$ is available, see [12] and Part I.

The reason is that the multiplier $(1+z) / 2$ cancels the singularity of $q_{\mathrm{ref}}$ at the point $z=-1 \in \bar{D}_{1}$; see the next lemma. (Recall that form (3.16) is also more suitable for the stable implementation of the discrete TBC, see [12].)

LEMma 3.3. The functions

$$
\nu_{1}(z):=\gamma(z)+\sqrt{\gamma^{2}(z)-1}, \quad \nu_{2}(z):=\gamma(z)-\sqrt{\gamma^{2}(z)-1}, \quad q_{\mathrm{ref}}=\frac{\nu_{1}-\nu_{2}}{2 h}
$$

have unique continuous extensions $\nu_{1 e}, \nu_{2 e}, q_{\mathrm{ref}, e}=\left(\nu_{1 e}-\nu_{2 e}\right) /(2 h)$ from $D_{1}$ to $\bar{D}_{1} \backslash\{-1\}$.

Moreover the functions

$$
\frac{1+z}{2} \nu_{1 e}(z), \frac{1+z}{2} \nu_{2 e}(z), \frac{1+z}{2} q_{\mathrm{ref}, e}(z),
$$

defined respectively by $0,-2 i a_{1}, i a_{1} / h$ for $z=-1$, are continuous on $\bar{D}_{1}$.

Proof. The function $\sqrt{\zeta^{2}-1}$ can be extended from $\{\operatorname{Im} \zeta<0\}$ by a function

$-\sqrt{\zeta^{2}-1}$, continuous on $\{\operatorname{Im} \zeta \leqslant 0\}$, by setting for real $x$

$$
\sqrt{\zeta^{2}-1} e_{e}:=\lim _{\varepsilon \rightarrow 0_{+}} \sqrt[-]{(x-i \varepsilon)^{2}-1}=\left\{\begin{array}{l}
i \sqrt{1-x^{2}} \text { for }|x| \leqslant 1, \\
-(\operatorname{sgn} x) \sqrt{x^{2}-1} \text { for }|x|>1 .
\end{array}\right.
$$

This implies the first part of the lemma. Note that $0<\left|\nu_{1 e}(z)\right| \leqslant 1$ and $\left|\nu_{2 e}(z)\right| \geqslant 1$ on $\bar{D}_{1}$.

The limit relations $((1+z) / 2) \nu_{1 e}(z) \rightarrow 0$ and $((1+z) / 2)\left(\nu_{1 e}(z)+\nu_{2 e}(z)\right)=(1+$ $z) \gamma(z) \rightarrow-2 i a_{1}$ as $z \rightarrow-1$ and $z \in \bar{D}_{1} \backslash\{-1\}$ yield the second part of the lemma.

Let $H^{\infty}\left(D_{r}\right)$ be the Hardy space of functions $p$ analytic in $D_{r}$ such that $\sup _{D_{r}}|p(z)|<\infty$. For such functions the trace $\left.p\right|_{\partial D_{r}} \in L^{\infty}\left(\partial D_{r}\right)$ is well defined; moreover, $\sup _{D_{r}}|p(z)|=\|p\|_{L^{\infty}\left(\partial D_{r}\right)}[18]$.

Proposition 3.4. Let $q \in H^{\infty}\left(D_{r}\right)$ for $0<r<1$ or $(1+z) q(z) \in H^{\infty}\left(D_{1}\right)$ for $r=1$. Then the following bound holds, for $\mathcal{E}^{m} \equiv r^{-m}$ :

$$
\sup _{M \geqslant 1}\left\|\mathcal{S}_{\mathrm{ref}}-\mathcal{S}\right\|_{M, \mathcal{E}} \leqslant\left\|\frac{1+z}{2} q_{\mathrm{ref}, e}(z)-\frac{1+z}{2} q(z)\right\|_{L^{\infty}\left(\partial D_{r}\right)} .
$$

Proof. For fixed $M \geqslant 1$ and $\Phi^{0}=0, \Phi^{1}, \ldots, \Phi^{M}$, we set $\Phi^{m}:=0$ for $m>M$. With an argument similar to that used in the proof of (3.6) applied to (3.16), we obtain the relations, for $r<1$ and $\mathcal{E}^{m} \equiv r^{-m}$

$$
\begin{aligned}
\left\|\frac{1}{\mathcal{E}}\left(\mathcal{S}_{\mathrm{ref}}-\mathcal{S}\right) \boldsymbol{\Phi}\right\|_{\omega_{M}^{\tau}}^{2} & =\tau \sum_{m=0}^{\infty}\left|\left(\frac{R_{\mathrm{ref}}}{\mathcal{E}}-\frac{R}{\mathcal{E}}\right)^{m} *\left(\frac{\Phi}{\mathcal{E}}\right)^{m}\right|^{2} \\
& =\frac{\tau}{2 \pi} \int_{0}^{2 \pi}\left|\varkappa\left(r e^{i \varphi}\right)\right|^{2}\left|\mathcal{T}[\Phi]\left(r e^{i \varphi}\right)\right|^{2} d \varphi \\
& \leqslant\|\varkappa\|_{L^{\infty}\left(\partial D_{r}\right)}^{2} \frac{\tau}{2 \pi} \int_{0}^{2 \pi}\left|\mathcal{T}[\Phi]\left(r e^{i \varphi}\right)\right|^{2} d \varphi=\|\varkappa\|_{L^{\infty}\left(\partial D_{r}\right)}^{2}\left\|\frac{\Phi}{\mathcal{E}}\right\|_{\omega_{M}^{\tau}}^{2},
\end{aligned}
$$


where

$$
\varkappa(z):=\frac{1+z}{2} q_{\mathrm{ref}, e}(z)-\frac{1+z}{2} q(z) .
$$

Here we have applied twice the Parseval equality; note also that $\mathcal{T}[\Phi]$ is simply a polynomial of degree not greater than $M$.

The case $r=1$ is reduced to the previous one by passing to the limit $r \rightarrow 1_{-}$and using Lemma 3.3 and the limit relation (see [18])

$$
\lim _{r \rightarrow 1_{-}}\|g\|_{L^{\infty}\left(\partial D_{r}\right)}=\|g\|_{L^{\infty}\left(\partial D_{1}\right)} \text { for } g \in H^{\infty}\left(D_{1}\right) .
$$

REMARK 3.2. In fact inequality (3.17) is an equality. To see that, we should analyze the unique inequality in the chain of relations (3.18) (which finally holds for any $0<r \leqslant 1)$ and show the inverse inequality

$C\left(\varkappa_{0}\right):=\sup _{M \geqslant 1} \sup _{\Phi} \frac{\int_{0}^{2 \pi} \varkappa_{0}(\varphi)\left|\mathcal{T}[\Phi]\left(r e^{i \varphi}\right)\right|^{2} d \varphi}{\int_{0}^{2 \pi}\left|\mathcal{T}[\Phi]\left(r e^{i \varphi}\right)\right|^{2} d \varphi} \geqslant\left\|\varkappa_{0}\right\|_{L^{\infty}(0,2 \pi)}$ for $\varkappa_{0}(\varphi):=\left|\varkappa\left(r e^{i \varphi}\right)\right|^{2}$,

where the second supremum is taken over all $\Phi: \bar{\omega}^{\tau} \rightarrow \mathbb{C}, \Phi^{m}=0$ for $m=0$ and $m>M$, and $\Phi \not \equiv 0$. Similarly to the proof of Claim 1 in Proposition 3.1, we get

$$
\begin{aligned}
C\left(\varkappa_{0}\right) & \geqslant \sup _{N \geqslant 1 T_{N} \neq 0} \frac{\int_{0}^{2 \pi} \varkappa_{0}\left|T_{N}\right|^{2} d \varphi}{\int_{0}^{2 \pi}\left|T_{N}\right|^{2} d \varphi} \\
& =\sup _{g \in L^{2}(0,2 \pi), g \neq 0} \frac{\int_{0}^{2 \pi} \varkappa_{0}|g|^{2} d \varphi}{\int_{0}^{2 \pi}|g|^{2} d \varphi}=\sup _{f \geqslant 0:\|f\|_{L^{1}(0,2 \pi)}=1} \int_{0}^{2 \pi} \varkappa_{0} f d \varphi .
\end{aligned}
$$

The first equality follows from the density of the set of all trigonometric polynomials in $L^{2}(0,2 \pi)$, and the second equality is obvious. Since $\varkappa_{0} \geqslant 0$, the last supremum (over f) equals $\left\|\varkappa_{0}\right\|_{L^{\infty}(0,2 \pi)}$, which completes the proof.

Notice that the right-hand side of (3.17) does not change if one replaces $\|\cdot\|_{L^{\infty}\left(D_{r}\right)}$ by $\|\cdot\|_{L^{\infty}\left(\partial D_{r}\right)}$.

Keeping in mind Remark 2.6, we can weaken the $L^{\infty}\left(\partial D_{r}\right)$-norm in the last proposition at the cost of a new multiplier unbounded in $\tau$.

Proposition 3.5. Let $1 \leqslant p \leqslant 2 \leqslant p_{1} \leqslant \infty$ and $1 / s=1 / p-1 / p_{1}$. Assume that $q \in$ $H^{s}\left(D_{r}\right)$ for some $0<r<1$ or $(1+z) q(z) \in H^{s}\left(D_{1}\right)$ for $r=1$. Then the following bound holds, for $\mathcal{E}^{m} \equiv r^{-m}$

$$
\sup _{M \geqslant 1}\left\|\mathcal{S}_{\mathrm{ref}}-\mathcal{S}\right\|_{M, \mathcal{E}, p, p_{1}} \leqslant \frac{1}{(2 \pi \tau)^{1 / s}}\left\|\frac{1+z}{2}\left(q_{\mathrm{ref}, e}(z)-q(z)\right)\right\|_{L^{s}\left(\partial D_{r}\right)} .
$$

Remark 3.3. For $p=p_{1}=2$, one has $s=\infty$ and the bound coincides with (3.17). On the other hand, for $p=1$ and $p_{1}=\infty$, one gets the minimal value $s=1$.

Proof. The argument is similar to the one used in Proposition 3.4, except that one exploits the Hausdorff-Young inequalities for series (for example see [11]) together with the Hölder inequality instead of the Parseval equality. 
Namely, for $r<1$ and $\mathcal{E}^{m} \equiv r^{-m}$, the following relations hold:

$$
\begin{aligned}
\left\|\frac{1}{\mathcal{E}}\left(\mathcal{S}-\mathcal{S}_{\mathrm{ref}}\right) \boldsymbol{\Phi}\right\|_{L^{p_{1}\left(\omega_{M}^{\tau}\right)}} & =\tau^{1 / p_{1}}\left(\sum_{m=0}^{\infty}\left|\left(\frac{R_{\mathrm{ref}}}{\mathcal{E}}-\frac{R}{\mathcal{E}}\right)^{m} *\left(\frac{\Phi}{\mathcal{E}}\right)^{m}\right|^{p_{1}}\right)^{1 / p_{1}} \\
& \leqslant \tau^{1 / p_{1}} \frac{1}{(2 \pi)^{1 / p_{1}^{\prime}}}\|\varkappa \mathcal{T}[\Phi]\|_{L^{p_{1}^{\prime}\left(\partial D_{r}\right)}} \\
& \leqslant \tau^{1 / p_{1}} \frac{1}{(2 \pi)^{s}}\|\varkappa\|_{L^{s}\left(\partial D_{r}\right)} \frac{1}{(2 \pi)^{1 / p^{\prime}}}\|\mathcal{T}[\Phi](z)\|_{L^{p^{\prime}}\left(\partial D_{r}\right)} \\
& \leqslant \tau^{1 / p_{1}} \frac{1}{(2 \pi)^{1 / s}}\|\varkappa\|_{L^{s}\left(\partial D_{r}\right)}\left(\sum_{m=0}^{\infty}\left|\frac{\Phi^{m}}{\mathcal{E}^{m}}\right|^{p}\right)^{1 / p} \\
& =\frac{\tau^{1 / p_{1}-1 / p}}{(2 \pi)^{1 / s}}\|\varkappa\|_{L^{s}\left(\partial D_{r}\right)}\left\|\frac{\Phi}{\mathcal{E}}\right\|_{L^{p}\left(\omega_{M}^{\tau}\right)}
\end{aligned}
$$

where $\left(\sum_{m=0}^{\infty}|Y|^{p_{1}}\right)^{1 / p_{1}}$, for $p_{1}=\infty$, is understood as $\sup _{m \geqslant 0}\left|Y^{m}\right|$. This implies the result for $r<1$.

The case $r=1$ is reduced once again to the previous one by passing to the limit as $r \rightarrow 1_{-}$with the help of the limit relation (see [18])

$$
\lim _{r \rightarrow 1_{-}}\|g\|_{L^{s}\left(\partial D_{r}\right)}=\|g\|_{L^{s}\left(\partial D_{1}\right)} \text { for } g \in H^{s}\left(D_{1}\right) .
$$

\section{The $2 \mathrm{D}$ case}

We recall that for applications in low-energy nuclear fission dynamics, the 1D model is oversimplified. In this section, we show how the above $1 \mathrm{D}$ results can be extended and exploited for a 2D situation of physical interest; see [7, 13]. So we turn to the generalized time-dependent 2D Schrödinger equation

$$
\begin{gathered}
i \hbar \frac{\partial \psi}{\partial t}=\mathcal{H} \psi \\
:=-\frac{\hbar^{2}}{2}\left[\frac{\partial}{\partial x}\left(B_{11} \frac{\partial \psi}{\partial x}\right)+\frac{\partial}{\partial x}\left(B_{12} \frac{\partial \psi}{\partial y}\right)+\frac{\partial}{\partial y}\left(B_{21} \frac{\partial \psi}{\partial x}\right)+\frac{\partial}{\partial y}\left(B_{22} \frac{\partial \psi}{\partial y}\right)\right]+V \psi \\
\text { for }(x, y) \in \Omega:=(0, \infty) \times(0, Y), \text { and } t>0
\end{gathered}
$$

involving the $2 \mathrm{D}$ Hamiltonian operator $\mathcal{H}$ with the real matrix $\left\{B_{p q}(x, y)\right\}_{p, q=1}^{2}$ that is symmetric and has eigenvalues not less than $\nu>0$ uniformly in $\Omega . V(x, y)$ is real in $\Omega$.

Equation (4.1) is supplemented with the following boundary condition, the condition at infinity and the initial condition

$$
\begin{aligned}
\left.\psi\right|_{\partial \Omega} & =0, \quad\|\psi(x, \cdot, t)\|_{L^{2}(0, Y)} \rightarrow 0 \text { as } x \rightarrow \infty, \text { for any } t>0, \\
\left.\psi\right|_{t=0} & =\psi^{0}(x, y) \text { in } \Omega .
\end{aligned}
$$

We assume that for some $X_{0}>0$,

$$
\begin{aligned}
B_{11}(x, y) & =B_{1 \infty}>0, \quad B_{12}(x, y)=B_{21}(x, y)=0, \quad B_{22}(x, y)=B_{2 \infty}>0, \\
V(x, y) & =V_{\infty} \text { and } \psi^{0}(x, y)=0 \text { for } x \geqslant X_{0}, \quad y \in[0, Y] .
\end{aligned}
$$


An explicit TBC for this problem is written for example in Part I.

In addition to the notation from Section 2, we define two mesh averaging operators with respect to $x$,

$$
\bar{s}_{x} W_{j}=\frac{W_{j-1}+W_{j}}{2}, \widehat{s}_{x} W_{j}:=\frac{h_{j} W_{j}+h_{j+1} W_{j+1}}{2 h_{j+1 / 2}} .
$$

We also introduce a mesh $\bar{\omega}_{\delta}$ in $y$ on $[0, Y]$ with the nodes $0=y_{0}<\cdots<y_{K}=Y$ and the steps $\delta_{k}:=y_{k}-y_{k-1}$. Let $\omega_{\delta}:=\bar{\omega}_{\delta} \backslash\{0, Y\}$. We define the backward and the modified forward difference quotients together with two mesh averaging operators with respect to $y$,

$$
\begin{aligned}
\bar{\partial}_{y} U_{k}:=\frac{U_{k}-U_{k-1}}{\delta_{k}}, \quad \widehat{\partial}_{y} U_{k}:=\frac{U_{k+1}-U_{k}}{\delta_{k+1 / 2}} \\
\bar{s}_{y} U_{k}=\frac{U_{k-1}+U_{k}}{2}, \quad \widehat{s}_{y} U_{k}:=\frac{\delta_{k} U_{k}+\delta_{k+1} U_{k+1}}{2 \delta_{k+1 / 2}},
\end{aligned}
$$

where $\delta_{k+1 / 2}:=\left(\delta_{k}+\delta_{k+1}\right) / 2$. We also need the inner products

$$
(U, W)_{\omega_{\delta}} \equiv(U, W)_{\stackrel{\circ}{H\left(\bar{\omega}_{\delta}\right)}}:=\sum_{k=1}^{K-1} U_{k} W_{k}^{*} \delta_{k+1 / 2}, \quad(U, W)_{\widetilde{\omega}_{\delta}}:=\sum_{k=1}^{K} U_{k} W_{k}^{*} \delta_{k}
$$

and the associated norms $\|\cdot\|_{\omega_{\delta}}$ and $\|\cdot\|_{\widetilde{\omega}_{\delta}}$, where $\stackrel{\circ}{H}\left(\bar{\omega}_{\delta}\right)$ is the space of functions $U$ : $\bar{\omega}_{\delta} \rightarrow \mathbb{C}$ with $\left.U\right|_{k=0, K}=0$.

We define the product meshes $\bar{\omega}_{\mathbf{h}, \infty}:=\bar{\omega}_{h, \infty} \times \bar{\omega}_{\delta}$ on $\bar{\Omega}, \bar{\omega}_{\mathbf{h}}:=\bar{\omega}_{h} \times \bar{\omega}_{\delta}$ on $[0, X] \times$ $[0, Y]$ and $\omega_{\mathbf{h}}:=\omega_{h} \times \omega_{\delta}$ and introduce two mesh inner products

$$
\begin{aligned}
(U, W)_{\omega_{\mathbf{h}}} & =(U, W)_{\omega_{h} \times \omega_{\delta}} \\
& :=\sum_{k=1}^{K-1}\left(U_{j k}, W_{j k}\right)_{\omega_{h}} \delta_{k+1 / 2}, \quad(U, W)_{\bar{\omega}_{\mathbf{h}}}:=\sum_{k=1}^{K-1}\left(U_{j k}, W_{j k}\right)_{\bar{\omega}_{h}} \delta_{k+1 / 2}
\end{aligned}
$$

together with the associated mesh norms $\|\cdot\|_{\omega_{\mathbf{h}}}$ and $\|\cdot\|_{\bar{\omega}_{\mathbf{h}}}$. The additional inner products $(\cdot, \cdot)_{\widetilde{\omega}_{h} \times \omega_{\delta}},(\cdot, \cdot)_{\omega_{h} \times \widetilde{\omega}_{\delta}}$ and $(\cdot, \cdot)_{\omega_{\delta} \times \omega_{M}^{\tau}}$ and the associated norms are defined similarly to the former one.

We continue to exploit the 2D mesh Hamiltonian operator, see [22] and Part I,

$$
\begin{aligned}
\mathcal{H}_{\mathbf{h}} W:= & -\frac{\hbar^{2}}{2}\left\{\widehat{\partial}_{x}\left(B_{11 h} \bar{\partial}_{x} W\right)+\widehat{\partial}_{x} \widehat{s}_{y}\left(B_{12 h} \bar{s}_{x} \bar{\partial}_{y} W\right)\right. \\
& \left.+\widehat{s}_{x} \widehat{\partial}_{y}\left(B_{21 h} \bar{\partial}_{x} \bar{s}_{y} W\right)+\widehat{\partial}_{y}\left(B_{22 h} \bar{\partial}_{y} W\right)\right\}+V_{h} W
\end{aligned}
$$

where the coefficients are given by the formulas

$$
B_{11 h}=\widehat{s}_{y} \widetilde{B}_{11}, \quad B_{12 h}=B_{21 h}=\widetilde{B}_{12}, \quad B_{22 h}=\widehat{s}_{x} \widetilde{B}_{22}, \quad V_{h}=\widehat{s}_{x} \widehat{s}_{y} \widetilde{V}
$$

with $\widetilde{B}_{j k}:=B\left(x_{j}-h_{j} / 2, y_{k}-\delta_{k} / 2\right)$. We study the following Crank-Nicolson finite- 
difference with general 2D approximate TBC, see Part I

$$
\begin{aligned}
i \hbar \bar{\partial}_{t} \Psi= & \mathcal{H}_{\mathbf{h}} \bar{s}_{t} \Psi+F \text { on } \omega_{\mathbf{h}} \times \omega^{\tau}, \\
\left.\Psi^{m}\right|_{j=0}= & 0,\left.\quad \Psi^{m}\right|_{k=0, K}=0 \text { for } m \geqslant 1, \\
& {\left.\left[\frac{\hbar^{2}}{2} B_{1 \infty} \bar{\partial}_{x} \bar{s}_{t} \Psi-\frac{h}{2}\left(i \hbar \bar{\partial}_{t} \Psi+\frac{\hbar^{2}}{2} B_{2 \infty} \widehat{\partial}_{y} \bar{\partial}_{y} \bar{s}_{t} \Psi-V_{\infty} \bar{s}_{t} \Psi\right)\right]^{m}\right|_{j=J} } \\
= & \frac{\hbar^{2}}{2} B_{1 \infty} \mathcal{S}^{m} \Psi_{J}^{m}+\hat{G}^{m} \text { on } \omega_{\delta} \text { for } m \geqslant 1, \\
& \Psi^{0}=\Psi_{\mathbf{h}}^{0} \text { on } \bar{\omega}_{\mathbf{h}},
\end{aligned}
$$

where $\Psi_{\mathbf{h} j k}^{0}=\psi^{0}\left(x_{j}, y_{k}\right)$ (for definiteness) and thus $\left.\Psi_{\mathbf{h}}^{0}\right|_{j=J}=0$; we assume that the two conjunction conditions $\left.\Psi_{\mathbf{h}}^{0}\right|_{j=0}=0$ and $\left.\Psi_{\mathbf{h}}^{0}\right|_{k=0, K}=0$ are also valid. Hereafter we exploit collections $\Psi_{J}^{m}=\left\{\Psi_{J}^{n}\right\}_{n=0}^{m}$ and $\Psi_{j}^{m}=\left\{\Psi_{j k}^{m}\right\}_{k=0}^{K}, j \geqslant 0$ and $m \geqslant 0$; recall that $\mathcal{S}^{m} \mathbf{\Psi}_{J}^{m}$ is non-local not only in time as in the $1 \mathrm{D}$ case but in $y$ as well. Once more $F: \omega_{\mathbf{h}} \times \omega^{\tau} \rightarrow \mathbb{C}$ and $\hat{G}: \omega_{\delta} \times \omega^{\tau} \rightarrow \mathbb{C}$ are given perturbations that are introduced to study the stability of the scheme.

Proposition 4.1. Let $\Psi$ be a solution of the finite-difference scheme (4.4)-(4.7) with any $\Psi_{\mathbf{h}}^{0}$ such that $\left.\Psi_{\mathbf{h}}^{0}\right|_{j=0, J}=0$ and $\left.\Psi_{\mathbf{h}}^{0}\right|_{k=0, K}=0$. Assume that the operator $\mathcal{S}$ satisfies the inequality

$$
\operatorname{Im}\left(\frac{1}{E^{2}} \mathcal{S} \Phi, \bar{s}_{t} \Phi\right)_{\omega_{\delta} \times \omega_{M}^{\tau}} \geqslant 0 \text { for any } M \geqslant 1,
$$

for some $E \in N D\left(\bar{\omega}^{\tau}\right)$ and any function $\Phi: \bar{\omega}_{\delta} \times \bar{\omega}^{\tau} \rightarrow \mathbb{C}$ such that $\Phi^{0}=0$ and $\left.\Phi\right|_{k=0, K}=0$. Then the following stability bound holds, for any $M \geqslant 1$

$$
\begin{aligned}
& \max _{0 \leqslant m \leqslant M}\left\|\Psi^{m}\right\|_{\bar{\omega}_{\mathbf{h}}} \\
\leqslant & E^{M}\left(\left\|\Psi_{\mathbf{h}}^{0}\right\|_{\bar{\omega}_{\mathbf{h}}}+\frac{2}{\hbar} \sum_{m=1}^{M} \frac{1}{E^{m}}\left\|F^{m}\right\|_{\omega_{\mathbf{h}}} \tau_{m}+\frac{2}{\hbar} \sqrt{\frac{2}{h}} \sum_{m=1}^{M} \frac{1}{E^{m}}\left\|\hat{G}^{m}\right\|_{\omega_{\delta}} \tau_{m}\right) .
\end{aligned}
$$

Proof. From the proof of Proposition 4.1 in Part I it follows that

$$
\frac{\hbar}{2} \bar{\partial}_{t}\|\Psi\|_{\bar{\omega}_{\mathbf{h}}}^{2}=\operatorname{Im}\left\{\left(F, \bar{s}_{t} \Psi\right)_{\omega_{\mathbf{h}}}-\left(\hat{G}, \bar{s}_{t} \Psi_{J}\right)_{\omega_{\delta}}-\frac{\hbar^{2}}{2} B_{1 \infty}\left(\mathcal{S} \Psi_{J}, \bar{s}_{t} \Psi_{J}\right)_{\omega_{\delta}}\right\} .
$$

Similarly to the proof of Proposition 2.1, for $Y=\Psi / E$, the relations

$$
\begin{aligned}
& \frac{\hbar}{2}\left[\bar{\partial}_{t}\|Y\|_{\omega_{\mathbf{h}}}^{2}+\frac{\bar{\partial}_{t}\left(E^{2}\right)}{E^{2}}\|\check{Y}\|_{\bar{\omega}_{\mathbf{h}}}^{2}\right] \\
= & \operatorname{Im}\left\{\left(F, \frac{1}{E^{2}} \bar{s}_{t}(E Y)\right)_{\omega_{\mathbf{h}}}-\left(\hat{G}, \frac{1}{E^{2}} \bar{s}_{t}\left(E Y_{J}\right)\right)_{\omega_{\delta}}-\frac{\hbar^{2}}{2 E^{2}} B_{1 \infty}\left(\mathcal{S} \Psi_{J}, \bar{s}_{t} \Psi_{J}\right)_{\omega_{\delta}}\right\} \\
\leqslant & \|F\|_{\omega_{\mathbf{h}}} \frac{1}{E} \bar{s}_{t}\|Y\|_{\omega_{\mathbf{h}}}+\|\hat{G}\|_{\omega_{\delta}} \frac{1}{E} \bar{s}_{t}\left\|Y_{J}\right\|_{\omega_{\delta}}-\frac{\hbar^{2}}{2 E^{2}} B_{1 \infty} \operatorname{Im}\left(\mathcal{S} \Psi_{J}, \bar{s}_{t} \Psi_{J}\right)_{\omega_{\delta}}
\end{aligned}
$$


and consequently the bound

$$
\max _{0 \leqslant m \leqslant M}\left\|Y^{m}\right\|_{\bar{\omega}_{\mathbf{h}}} \leqslant\left\|Y^{0}\right\|_{\bar{\omega}_{h}}+\frac{2}{\hbar} \sum_{m=1}^{M} \frac{1}{E^{m}}\left\|F^{m}\right\|_{\omega_{\mathbf{h}}} \tau_{m}+\frac{2}{\hbar} \sqrt{\frac{2}{h}} \sum_{m=1}^{M} \frac{1}{E^{m}}\left\|\hat{G}^{m}\right\|_{\omega_{\delta}} \tau_{m}
$$

hold, and the result follows.

REMARK 4.1. The quantity $E^{M}\left[\hbar B_{1 \infty} \operatorname{Im}\left(\frac{1}{E^{2}} \mathcal{S} \Psi_{J}, \bar{s}_{t} \Psi_{J}\right)_{\omega_{\delta} \times \omega_{M}^{\tau}}\right]^{1 / 2}$ is also bounded by the right-hand side of (4.9), for any $M \geqslant 1$.

COROLlary 4.2. Let the hypotheses of Proposition 4.1 together with the a priori estimate

$$
\left\|\bar{\partial}_{x} \Psi\right\|_{\widetilde{\omega}_{h} \times \omega_{\delta}} \leqslant C_{0} \hat{E} \quad \text { on } \bar{\omega}^{\tau},
$$

for some $\hat{E} \in N D\left(\bar{\omega}^{\tau}\right)$, be valid. Then the $\hat{G}$-term in bound (4.9) can be replaced by

$$
\left[\frac{2 \sqrt{2 C_{0}}}{\hbar} \sum_{m=1}^{M} \frac{\sqrt{\bar{s}_{t} \hat{E}^{m}}}{E^{m}}\left\|\hat{G}^{m}\right\|_{\omega_{\delta}} \tau_{m}\right]^{2 / 3} .
$$

The proof is similar to one for Corollary 2.2 and exploits the multiplicative inequality

$$
\max _{0 \leqslant j \leqslant J}\left\|W_{j k}\right\|_{\omega_{\delta}}^{2} \leqslant 2\|W\|_{\bar{\omega}_{\mathbf{h}}}\left\|\bar{\partial}_{x} W\right\|_{\widetilde{\omega}_{h} \times \omega_{\delta}},
$$

where $\left.W\right|_{j=0}=0$ (which follows from (2.16)).

We introduce the 2D Hermitian-symmetric bilinear form

$$
\begin{aligned}
\mathcal{L}_{\bar{\omega}_{\mathbf{h}}}(U, W):= & \frac{\hbar^{2}}{2} \sum_{j=1}^{J} \sum_{k=1}^{K}\left\{\widetilde{B}_{11} \bar{s}_{y}\left[\left(\bar{\partial}_{x} U\right) \bar{\partial}_{x} W^{*}\right]+\widetilde{B}_{12}\left(\bar{s}_{x} \bar{\partial}_{y} U\right) \bar{\partial}_{x} \bar{s}_{y} W^{*}\right. \\
& \left.+\widetilde{B}_{21}\left(\bar{\partial}_{x} \bar{s}_{y} U\right) \bar{s}_{x} \bar{\partial}_{y} W^{*}+\widetilde{B}_{22} \bar{s}_{x}\left[\left(\bar{\partial}_{y} U\right) \bar{\partial}_{y} W^{*}\right]\right\}_{j k} h_{j} \delta_{k}+\left(V_{h} U, W\right)_{\bar{\omega}_{\mathbf{h}}}
\end{aligned}
$$

for $U, W: \bar{\omega}_{\mathbf{h}} \rightarrow \mathbb{C}$. The identity

$$
\begin{aligned}
& \mathcal{L}_{\bar{\omega}_{\mathbf{h}}}(U, W) \\
= & \left(\mathcal{H}_{\mathbf{h}} U, W\right)_{\omega_{\mathbf{h}}}+\left(\left\{\frac{\hbar^{2}}{2} B_{1 \infty} \bar{\partial}_{x} U-\frac{h}{2}\left(\frac{\hbar^{2}}{2} B_{2 \infty} \widehat{\partial}_{y} \bar{\partial}_{y} U-V_{\infty} U\right)\right\}_{J}, W\right)_{\omega_{\delta}}
\end{aligned}
$$

holds provided that $\left.W\right|_{j=0}=0$ and $\left.W\right|_{k=0, K}=0$; actually this has already been applied in the proof of Proposition 4.1 in Part I. Moreover, for such $W$, taking into account the assumptions on $\left\{B_{p q}\right\}_{p, q=1}^{2}$ and inequalities like $\bar{s}_{y}\left(|U|^{2}\right) \geqslant\left|\bar{s}_{y} U\right|^{2}$, we also get the inequality [22]

$$
\begin{aligned}
\mathcal{L}_{\bar{\omega}_{\mathbf{h}}}(W, W) & \geqslant \nu \frac{\hbar^{2}}{2} \sum_{j=1}^{J} \sum_{k=1}^{K}\left\{\bar{s}_{y}\left(\left|\bar{\partial}_{x} W\right|^{2}\right)+\bar{s}_{x}\left(\left|\bar{\partial}_{y} W\right|^{2}\right)\right\}_{j k} h_{j} \delta_{k}+\left(V_{h} W, W\right)_{\bar{\omega}_{\mathbf{h}}} \\
& =\nu_{1}\left(\left\|\bar{\partial}_{x} W\right\|_{\widetilde{\omega}_{h} \times \omega_{\delta}}^{2}+\left\|\bar{\partial}_{y} W\right\|_{\omega_{h} \times \widetilde{\omega}_{\delta}}^{2}\right)+\left(V_{h} W, W\right)_{\bar{\omega}_{\mathbf{h}}}
\end{aligned}
$$


and consequently

$$
\|W\|_{\mathcal{H}_{\mathbf{h}}+v I ; \bar{\omega}_{\mathbf{h}}}^{2}:=\mathcal{L}_{\bar{\omega}_{\mathbf{h}}}(W, W)+v\|W\|_{\bar{\omega}_{\mathbf{h}}}^{2} \geqslant \nu_{1}\left(\left\|\bar{\partial}_{x} W\right\|_{\widetilde{\omega}_{h} \times \omega_{\delta}}^{2}+\left\|\bar{\partial}_{y} W\right\|_{\omega_{h} \times \widetilde{\omega}_{\delta}}^{2}\right),
$$

for any real number $v \geqslant v_{0}$ with $v_{0}:=-\min _{\left(\bar{\omega}_{h} \backslash\{0\}\right) \times \omega_{\delta}} V_{h}$.

Proposition 4.3. Let $\Psi$ be a solution of the finite-difference scheme (4.4)-(4.7) with any $\Psi_{\mathbf{h}}^{0}$ such that $\left.\Psi_{\mathbf{h}}^{0}\right|_{j=0, J}=0$ and $\left.\Psi_{\mathbf{h}}^{0}\right|_{k=0, K}=0$ and $\hat{G}=0$. Assume that the operator $\mathcal{S}$ satisfies the inequality

$$
\operatorname{Im}\left(\mathcal{S} \Phi, i \hbar \bar{\partial}_{t} \Phi+v \bar{s}_{t} \Phi\right)_{\omega_{\delta} \times \omega_{M}^{\tau}} \geqslant 0 \text { for any } M \geqslant 1
$$

for any $\Phi: \bar{\omega}_{\delta} \times \bar{\omega}^{\tau} \rightarrow \mathbb{C}$ such that $\Phi^{0}=0$ and $\left.\Phi\right|_{k=0, K}=0$ and some $v \geqslant v_{0}$. Then the following bound holds

$$
\max _{0 \leqslant m \leqslant M}\left\|\Psi^{m}\right\|_{\mathcal{H}_{\mathbf{h}}+v I ; \bar{\omega}_{\mathbf{h}}} \leqslant\left\|\Psi_{\mathbf{h}}^{0}\right\|_{\mathcal{H}_{\mathbf{h}}+v I ; \bar{\omega}_{\mathbf{h}}}+\frac{2}{\hbar} \sum_{m=1}^{M}\left\|F^{m}\right\|_{\mathcal{H}_{\mathbf{h}}+v I ; \bar{\omega}_{\mathbf{h}}} \tau_{m} \text { for any } \quad M \geqslant 1 ;
$$

hereafter $F_{j k}^{m}$ is extended by zero for both $j=0, J$ and $k=0, K$.

Proof. Similarly to the proof of Proposition 2.3, taking the $(\cdot, \cdot)_{\omega_{h}}$-inner product of equation (4.4) and $\mathcal{H}_{\mathbf{h}} \bar{s}_{t} \Psi$ and applying identity (4.11), we get

$$
\begin{aligned}
& \mathcal{L}_{\bar{\omega}_{\mathbf{h}}}\left(i \hbar \bar{\partial}_{t} \Psi, \bar{s}_{t} \Psi\right)-\left(i \hbar \bar{\partial}_{t} \Psi, \frac{\hbar^{2}}{2} B_{1 \infty} \bar{\partial}_{x} \bar{s}_{t} \Psi_{J}-\frac{h}{2}\left(\frac{\hbar^{2}}{2} B_{2 \infty} \widehat{\partial}_{y} \bar{\partial}_{y} \bar{s}_{t} \Psi_{J}-V_{\infty} \bar{s}_{t} \Psi_{J}\right)\right)_{\omega_{\delta}} \\
& =\left\|\mathcal{H}_{\mathbf{h}} \bar{s}_{t} \Psi\right\|_{\omega_{\mathbf{h}}}^{2}+\left(F, \mathcal{H}_{\mathbf{h}} \bar{s}_{t} \Psi\right)_{\omega_{\mathbf{h}}} .
\end{aligned}
$$

Notice that

$$
\operatorname{Re} \mathcal{L}_{\bar{\omega}_{\mathbf{h}}}\left(\bar{\partial}_{t} \Psi, \bar{s}_{t} \Psi\right)=\frac{1}{2} \bar{\partial}_{t} \mathcal{L}_{\bar{\omega}_{\mathbf{h}}}(\Psi, \Psi)
$$

and, by virtue of the boundary condition (4.6),

$$
\left\{\frac{\hbar^{2}}{2} B_{1 \infty} \bar{\partial}_{x} \bar{s}_{t} \Psi-\frac{h}{2}\left(\frac{\hbar^{2}}{2} B_{2 \infty} \widehat{\partial}_{y} \bar{\partial}_{y} \bar{s}_{t} \Psi-V_{\infty} \bar{s}_{t} \Psi\right)\right\}_{J}=\frac{h}{2} i \hbar \bar{\partial}_{t} \Psi_{J}+\frac{\hbar^{2}}{2} B_{1 \infty} \mathcal{S} \Psi_{J} .
$$

Consequently after taking the imaginary part of both sides of (4.15), we find

$$
\frac{\hbar}{2} \bar{\partial}_{t} \mathcal{L}_{\bar{\omega}_{\mathbf{h}}}(\Psi, \Psi)=\operatorname{Im}\left\{\left(F, \mathcal{H}_{\mathbf{h}} \bar{s}_{t} \Psi\right)_{\omega_{\mathbf{h}}}-\frac{\hbar^{2}}{2} B_{1 \infty}\left(\mathcal{S} \Psi_{J}, i \hbar \bar{\partial}_{t} \Psi_{J}\right)_{\omega_{\delta}}\right\}
$$

Combining this equality and (4.10), we obtain

$$
\begin{aligned}
& \frac{\hbar}{2} \bar{\partial}_{t}\left[\mathcal{L}_{\bar{\omega}_{\mathbf{h}}}(\Psi, \Psi)+v\|\Psi\|_{\bar{\omega}_{\mathbf{h}}}^{2}\right] \\
&=\operatorname{Im}\left\{\left(F,\left(\mathcal{H}_{\mathbf{h}}+v I\right) \bar{s}_{t} \Psi\right)_{\omega_{\mathbf{h}}}-\frac{\hbar^{2}}{2} B_{1 \infty}\left(\mathcal{S} \Psi_{J}, i \hbar \bar{\partial}_{t} \Psi_{J}+v \bar{s}_{t} \Psi\right)_{\omega_{\delta}}\right\} .
\end{aligned}
$$

The last equality implies the result. 
COROLlary 4.4. Let us consider the scheme (4.4)-(4.7) in the case $F=0$ and $\hat{G}=0$, for some $v \geqslant v_{0}$. Condition (4.13) for $\Phi=\Psi_{J}$ is valid if and only if the solution satisfies the equality

$$
\max _{m \geqslant 0}\left\|\Psi^{m}\right\|_{\mathcal{H}_{\mathbf{h}}+v I ; \bar{\omega}_{\mathbf{h}}}=\left\|\Psi_{\mathbf{h}}^{0}\right\|_{\mathcal{H}_{\mathbf{h}}+v I ; \bar{\omega}_{\mathbf{h}}}
$$

The result follows from equality (4.16).

Now we consider the Crank-Nicolson scheme on the infinite mesh for the original problem (4.1)-(4.3)

$$
\begin{aligned}
i \hbar \bar{\partial}_{t} \Psi & =\mathcal{H}_{\mathbf{h}} \bar{s}_{t} \Psi+F \text { on } \omega_{\mathbf{h}, \infty} \times \omega^{\tau}, \\
\left.\Psi^{m}\right|_{j=0} & =0,\left.\quad \Psi^{m}\right|_{k=0, K}=0 \text { for } m \geqslant 1, \\
\Psi^{0} & =\Psi_{\mathbf{h}}^{0} \text { on } \bar{\omega}_{\mathbf{h}, \infty},
\end{aligned}
$$

where $F$ is a given perturbation. We consider only the solutions having the property $\Psi^{m} \in H_{\mathbf{h}}$ for any $m \geqslant 0$, where $H_{\mathbf{h}}$ is a Hilbert space consisting of functions $W: \bar{\omega}_{\mathbf{h}, \infty} \rightarrow$ $\mathbb{C}$ such that $\left.W\right|_{j=0}=0,\left.W\right|_{k=0, K}=0$ and $\sum_{j=1}^{\infty}\left\|W_{j k}\right\|_{\omega_{\delta}}^{2}<\infty$, equipped with the inner product $(U, W)_{H_{\mathbf{h}}}:=\sum_{j=1}^{\infty}\left(U_{j k}, W_{j k}\right)_{\omega_{\delta}} h_{j+1 / 2}$.

We define the 2D Hermitian-symmetric bilinear form

$$
\begin{aligned}
\mathcal{L}_{\bar{\omega}_{\mathbf{h}, \infty}}(U, W):= & \frac{\hbar^{2}}{2} \sum_{j=1}^{\infty} \sum_{k=1}^{K}\left\{\widetilde{B}_{11} \bar{s}_{y}\left[\left(\bar{\partial}_{x} U\right) \bar{\partial}_{x} W^{*}\right]+\widetilde{B}_{12}\left(\bar{s}_{x} \bar{\partial}_{y} U\right) \bar{\partial}_{x} \bar{s}_{y} W^{*}\right. \\
& \left.+\widetilde{B}_{21}\left(\bar{\partial}_{x} \bar{s}_{y} U\right) \bar{s}_{x} \bar{\partial}_{y} W^{*}+\widetilde{B}_{22} \bar{s}_{x}\left[\left(\bar{\partial}_{y} U\right) \bar{\partial}_{y} W^{*}\right]\right\}_{j k} h_{j} \delta_{k}+\left(V_{h} U, W\right)_{H_{\mathbf{h}}} \\
= & \left(\mathcal{H}_{\mathbf{h}} U, W\right)_{H_{\mathbf{h}}}
\end{aligned}
$$

for $U, W \in H_{\mathbf{h}}$; we set $\left.\mathcal{H}_{\mathbf{h}} U\right|_{j=0}:=0$ and $\left.\mathcal{H}_{\mathbf{h}} U\right|_{k=0, K}:=0$. We have

$$
\|W\|_{\mathcal{H}_{\mathbf{h}}+v I}^{2}:=\mathcal{L}_{\bar{\omega}_{\mathbf{h}, \infty}}(W, W)+v\|W\|_{H_{\mathbf{h}}}^{2} \geqslant \nu_{1}\left(\sum_{j=1}^{\infty}\left\|\bar{\partial}_{x} W_{j k}\right\|_{\omega_{\delta}}^{2} h_{j}+\sum_{k=1}^{K}\left\|\bar{\partial}_{y} W_{j k}\right\|_{H_{h}}^{2} \delta_{k}\right),
$$

for any $v \geqslant v_{0}$, by passing to the limit as $j_{0} \rightarrow \infty$ in relations like (4.12) but for the $\operatorname{mesh}\left\{x_{j}\right\}_{j=0}^{j_{0}}$.

Proposition 4.5. Let $F^{m} \in H_{\mathbf{h}}$ for any $m \geqslant 1$ and $\Psi_{\mathbf{h}}^{0} \in H_{\mathbf{h}}$. There exists a unique solution to the scheme (4.17)-(4.19), and the following stability bound holds:

$$
\max _{0 \leqslant m \leqslant M}\left\|\Psi^{m}\right\|_{\mathcal{H}_{\mathbf{h}}+v I} \leqslant\left\|\Psi_{\mathbf{h}}^{0}\right\|_{\mathcal{H}_{\mathbf{h}}+v I}+\frac{2}{\hbar} \sum_{m=1}^{M}\left\|F^{m}\right\|_{\mathcal{H}_{\mathbf{h}}+v I} \tau_{m} \quad \text { for any } \quad M \geqslant 1
$$

for any $v \geqslant v_{0}$. Moreover, for $F=0$, the following conservation law holds:

$$
\left\|\Psi^{m}\right\|_{\mathcal{H}_{\mathbf{h}}+v I}=\left\|\Psi_{\mathbf{h}}^{0}\right\|_{\mathcal{H}_{\mathbf{h}}+v I} \text { for any } m \geqslant 0
$$


Proof. The result is similar to Proposition 2.5 and is derived from Proposition 4.3 in Part I and the two energy-type equalities

$$
\begin{aligned}
\frac{\hbar}{2} \bar{\partial}_{t}\|\Psi\|_{H_{\mathbf{h}}}^{2} & =\operatorname{Im}\left(F, \bar{s}_{t} \Psi\right)_{H_{\mathbf{h}}}, \\
\frac{\hbar}{2} \bar{\partial}_{t} \mathcal{L}_{\bar{\omega}_{\mathbf{h}, \infty}}(\Psi, \Psi) & =\operatorname{Im}\left(F, \mathcal{H}_{\mathbf{h}} \bar{s}_{t} \Psi\right)_{H_{\mathbf{h}}} .
\end{aligned}
$$

Corollary 4.6. Let $F^{m}=0$ and $\Psi_{\mathbf{h}}^{0}=0$ on $\bar{\omega}_{\mathbf{h}, \infty} \backslash \omega_{\mathbf{h}}$ for $m \geqslant 1$. If the solution of the scheme (4.17)-(4.19) satisfies the relation

$$
\left(\stackrel{\circ}{\partial}_{x} \bar{s}_{t} \Psi\right)_{J}^{m}=\mathcal{S}_{\mathrm{ref}}^{m} \boldsymbol{\Psi}_{J}^{m} \text { on } \omega_{\delta} \text { for any } m \geqslant 1,
$$

then the following equality holds, for any $M \geqslant 1$ and any real $v$ :

$$
\begin{aligned}
& \hbar B_{1 \infty} \operatorname{Im}\left(\mathcal{S}_{\mathrm{ref}} \Psi_{J}, i \hbar \bar{\partial}_{t} \Psi_{J}+v \bar{s}_{t} \Psi_{J}\right)_{\omega_{\delta} \times \omega_{M}^{\tau}} \\
= & \left(\frac{\hbar^{2}}{2} B_{2 \infty}\left\|\bar{\partial}_{y} \Psi_{J}^{M}\right\|_{\widetilde{\omega}_{\delta}}^{2}+\left(V_{\infty}+v\right)\left\|\Psi_{J}^{M}\right\|_{\omega_{\delta}}^{2}\right) \frac{h}{2} \\
& +\sum_{j=J+1}^{\infty}\left(\frac{\hbar^{2}}{2} B_{1 \infty}\left\|\bar{\partial}_{x} \Psi_{j}^{M}\right\|_{\omega_{\delta}}^{2}+\frac{\hbar^{2}}{2} B_{2 \infty}\left\|\bar{\partial}_{y} \Psi_{j}^{M}\right\|_{\widetilde{\omega}_{\delta}}^{2}+\left(V_{\infty}+v\right)\left\|\Psi_{j}^{M}\right\|_{\omega_{\delta}}^{2}\right) h .
\end{aligned}
$$

Consequently, $\mathcal{S}=\mathcal{S}_{\text {ref }}$ satisfies condition (4.13) for $\Phi=\Psi_{J}$ and any $v \geqslant-V_{\infty}$. Moreover, for the uniform mesh $\bar{\omega}^{\tau}, \mathcal{S}_{\text {ref }}$ is the operator of the $2 D$ discrete TBC, see [5] and Part $I$, and the condition is valid for any $\Phi$ mentioned in it.

Equality (4.23) follows from combined equalities (4.20) and (4.21) together with equality (4.16).

For the uniform mesh $\bar{\omega}^{\tau}$, it follows from Part I that any function $\Phi: \omega_{\delta} \times \omega_{M}^{\tau} \rightarrow \mathbb{C}$ can be extended as the solution of the scheme (4.17)-(4.19) such that $\Psi_{J}=\Phi$ on $\omega_{\delta} \times \omega_{M}^{\tau}$, with some $F$ such that $F^{m}=0$ on $\omega_{\mathbf{h}, \infty} \backslash \omega_{\mathbf{h}}$ for $m \geqslant 1$ and $\Psi_{\mathbf{h}}^{0}=0$. Namely, one can set $\boldsymbol{\Psi}_{j}=0$ for $0 \leqslant j \leqslant J-2$, extend $\Phi^{m}=0$ for $m>M$, and construct $\boldsymbol{\Psi}_{j}$ for $j \geqslant J-1$ as the solution of the equation

$$
i \hbar \bar{\partial}_{t} \Psi=\frac{\hbar^{2}}{2}\left(B_{1 \infty} \widehat{\partial}_{x} \bar{\partial}_{x}+B_{2 \infty} \widehat{\partial}_{y} \bar{\partial}_{y}\right) \bar{s}_{t} \Psi+V_{\infty} \bar{s}_{t} \Psi \quad \text { on } \quad\left(\omega_{\mathbf{h}, \infty} \backslash \omega_{\mathbf{h}}\right) \times \omega^{\tau}
$$

having the property $\Psi^{m} \in H_{\mathbf{h}}$ for any $m \geqslant 0$, with $\Psi^{0}=0$, exploiting the technique described in Part I for the derivation of the $2 \mathrm{D}$ discrete TBC. This completes the proof.

Let us go back to the scheme (4.4)-(4.7).

Proposition 4.7. Let the hypotheses of Proposition 4.3 be valid, with the following generalization of condition (4.13):

$$
\operatorname{Im}\left(\frac{1}{\hat{E}^{2}} \mathcal{S} \Phi, i \hbar \bar{\partial}_{t} \Phi+v \bar{s}_{t} \Phi\right)_{\omega_{\delta} \times \omega_{M}^{\tau}} \geqslant 0 \text { for any } M \geqslant 1
$$


for some $\hat{E} \in N D\left(\bar{\omega}^{\tau}\right)$, any $\Phi$ such as in (4.13) and some $v \geqslant v_{0}$. Then the following bound holds:

$$
\max _{0 \leqslant m \leqslant M}\left\|\Psi^{m}\right\|_{\mathcal{H}_{\mathbf{h}}+v I ; \bar{\omega}_{\mathbf{h}}} \leqslant \hat{E}^{M}\left(\left\|\Psi_{\mathbf{h}}^{0}\right\|_{\mathcal{H}_{\mathbf{h}}+v I ; \bar{\omega}_{\mathbf{h}}}+\frac{2}{\hbar} \sum_{m=1}^{M} \frac{1}{\hat{E}^{m}}\left\|F^{m}\right\|_{\mathcal{H}_{\mathbf{h}}+v I ; \bar{\omega}_{\mathbf{h}}} \tau_{m}\right),
$$

for any $M \geqslant 1$.

The proof is similar to that for Proposition 2.7 and follows from equality (4.16); see also the proof of Proposition 4.1.

Corollary 4.8. Under conditions of any of Propositions 4.1, 4.3 and 4.7, the scheme (4.4)-(4.7) is uniquely solvable provided that $\mathcal{S}$ is a linear operator.

Note that Proposition 2.9 remains valid for the scheme (4.4)-(4.7) provided that condition (2.31) is valid on $\omega_{\delta}$ for any $\Phi$ such as in (4.8) and any $m \geqslant 1$.

For any $\mathcal{E} \in N D\left(\bar{\omega}^{\tau}\right), p, p_{1} \in[1, \infty]$ and linear $\mathcal{S}$, we define the norm

$$
\|\mathcal{S}\|_{M, \mathcal{E}, p, p_{1}}:=\sup _{\Phi} \frac{\left\|\frac{1}{\mathcal{E}}\right\| \mathcal{S} \Phi\left\|_{\omega_{\delta}}\right\|_{L^{p_{1}\left(\omega_{M}^{\tau}\right)}}}{\left\|\frac{1}{\mathcal{E}}\right\| \Phi\left\|_{\omega_{\delta}}\right\|_{L^{p}\left(\omega_{M}^{\tau}\right)}},
$$

where supremum is taken over all $\Phi: \bar{\omega}_{\delta} \times \bar{\omega}_{M}^{\tau} \rightarrow \mathbb{C}, \Phi^{0}=0,\left.\Phi\right|_{k=0, K}=0$ and $\Phi \not \equiv 0$. In particular, for $p=p_{1}=2$

$$
\|\mathcal{S}\|_{M, \mathcal{E}, p, p_{1}}=\|\mathcal{S}\|_{M, \mathcal{E}}:=\sup _{\Phi} \frac{\left\|\frac{1}{\mathcal{E}} \mathcal{S} \Phi\right\|_{\omega_{\delta} \times \omega_{M}^{\tau}}}{\left\|\frac{1}{\mathcal{E}} \Phi\right\|_{\omega_{\delta} \times \omega_{M}^{\tau}}} .
$$

Let $\mathcal{S}_{\text {ref }}$ be a linear operator satisfying condition (4.8) for $E \equiv 1$ and $\Psi_{\text {ref }}$ be the solution of the scheme for $\mathcal{S}=\mathcal{S}_{\text {ref }}$.

Proposition 4.9. Let $F=0, G=0$ and $\mathcal{S}$ be linear. For any $\mathcal{E} \in N D\left(\bar{\omega}^{\tau}\right), M \geqslant 1$ and $p, p_{1} \in[1, \infty]$, the following claims are valid.

1. If condition (4.8) holds, then

$$
\begin{gathered}
\max _{0 \leqslant m \leqslant M}\left\|\Psi_{\mathrm{ref}}^{m}-\Psi^{m}\right\|_{\bar{\omega}_{\mathbf{h}}} \\
\leqslant \frac{2}{h} \hbar B_{1 \infty}\|\mathcal{E}\|_{L^{p_{1}^{\prime}\left(\omega_{M}^{\tau}\right)}}\left\|\frac{E}{\mathcal{E}}\right\|_{L^{p}\left(\omega_{M}^{\tau}\right)}\left\|\Psi_{\mathbf{h}}^{0}\right\|_{\bar{\omega}_{\mathbf{h}}}\left\|\mathcal{S}_{\text {ref }}-\mathcal{S}\right\|_{M, \mathcal{E}, p, p_{1}} .
\end{gathered}
$$

2. If condition (4.24) holds, then

$$
\begin{gathered}
\max _{0 \leqslant m \leqslant M}\left\|\Psi_{\mathrm{ref}}^{m}-\Psi^{m}\right\|_{\bar{\omega}_{\mathbf{h}}} \\
\leqslant \sqrt{\frac{2 X}{\nu_{1} h} \hbar B_{1 \infty}\|\mathcal{E}\|_{L^{p_{1}^{\prime}\left(\omega_{M}^{\tau}\right)} \|} \| \hat{E}}\left\|_{\mathcal{E}}\right\| \Psi_{L^{p}\left(\omega_{M}^{\tau}\right)}^{0}\left\|_{\mathcal{H}_{\mathbf{h}}+v I ; \bar{\omega}_{\mathbf{h}}}\right\| \mathcal{S}_{\text {ref }}-\mathcal{S} \|_{M, \mathcal{E}, p, p_{1}} .
\end{gathered}
$$

3. If both conditions (4.13) for $\mathcal{S}=\mathcal{S}_{\text {ref }}$ and condition (4.24) hold, then

$$
\max _{0 \leqslant m \leqslant M}\left\|\Psi_{\mathrm{ref}}^{m}-\Psi^{m}\right\|_{\bar{\omega}_{\mathbf{h}}}
$$




$$
\begin{gathered}
\leqslant \frac{1}{\sqrt{\nu_{1}}}\left(\sqrt{2 X} \hbar B_{1 \infty}\|\sqrt{\hat{E}+1} \mathcal{E}\|_{L^{p_{1}^{\prime}\left(\omega_{M}^{\tau}\right)}}\left\|\frac{\hat{E}}{\mathcal{E}}\right\|_{L^{p}\left(\omega_{M}^{\tau}\right)}\right)^{2 / 3}\left\|\Psi_{\mathbf{h}}^{0}\right\|_{\mathcal{H}_{\mathbf{h}}+v I ; \bar{\omega}_{\mathbf{h}}} \\
\times\left\|\mathcal{S}_{\mathrm{ref}}-\mathcal{S}\right\|_{M, \mathcal{E}, p, p_{1}}^{2 / 3} .
\end{gathered}
$$

The proof exploits Propositions 4.1, 4.3 and 4.7 and is quite similar to the proof of Proposition 2.10 and Remark 2.6; thus this is omitted. We also omit the counterpart of Remark 2.4.

In the sequel, we analyze one particular but important case of the $2 \mathrm{D}$ operators $\mathcal{S}$. Following Part I, we introduce the auxiliary mesh eigenvalue problem

$$
-\widehat{\partial}_{y} \bar{\partial}_{y} \Theta=\lambda \Theta \text { on } \omega_{\delta},\left.\quad \Theta\right|_{k=0, K}=0, \quad \Theta \not \equiv 0 .
$$

Let $\left\{\Theta_{l}, \lambda_{l}\right\}, 1 \leqslant l \leqslant K-1$, be its eigenpairs such that the functions $\left\{\Theta_{l}\right\}_{l=1}^{K-1}$ are real-valued and form an orthonormalized basis in $\stackrel{\circ}{H}\left(\bar{\omega}_{\delta}\right)$; recall that $\lambda_{l \delta}>0$ for all $l$. Clearly, for any $U \in \stackrel{\circ}{H}\left(\bar{\omega}_{\delta}\right)$, the expansion holds

$$
U=\mathcal{F}^{-1} U^{(\cdot)}:=\sum_{l=1}^{K-1} U^{(l)} \Theta_{l}
$$

with the coefficients

$$
U^{(l)}=(\mathcal{F} U)^{(l)}:=\left(U, \Theta_{l}\right)_{\omega_{\delta}} \text { for } 1 \leqslant l \leqslant K-1 .
$$

We consider $\mathcal{S}$ satisfying the property

$$
(\mathcal{F} \mathcal{S} \boldsymbol{\Phi})^{(l)}=\mathcal{S}_{l} \mathcal{F} \boldsymbol{\Phi}^{(l)} \text { for all } 1 \leqslant l \leqslant K-1,
$$

that is, admitting the representation

$$
\mathcal{S} \boldsymbol{\Phi}=\mathcal{F}^{-1}\left(\mathcal{S}_{l} \mathcal{F} \boldsymbol{\Phi}^{(l)}\right)
$$

with some 1D linear (for simplicity) operators $\mathcal{S}_{l}$, for the same $\Phi$ such as in (4.8) and (4.13).

We recall that (see Part I), for the uniform mesh $\bar{\omega}^{\tau}$, the operator $\mathcal{S}_{\text {ref }}$ of the 2D discrete TBC (see also Corollary 4.6), admits such a representation

$$
\mathcal{S}_{\text {ref }} \boldsymbol{\Phi}=\mathcal{F}^{-1}\left(\mathcal{S}_{\text {ref }, l} \mathcal{F} \boldsymbol{\Phi}^{(l)}\right)
$$

involving the $1 \mathrm{D}$ operators

$$
\mathcal{S}_{\mathrm{ref}, l} \boldsymbol{\Lambda}:=Q_{\mathrm{ref}, l} * \bar{s}_{t} \Lambda \text {, with } \quad Q_{\mathrm{ref}, l}:=\left.Q_{\mathrm{ref}}\right|_{V_{\infty}=V_{\infty l \delta}} \quad \text { and } \quad V_{\infty l \delta}:=V_{\infty}+\frac{\hbar^{2}}{2} B_{2 \infty} \lambda_{l \delta}
$$

Another form $\mathcal{S}_{\text {ref }, l} \Lambda:=R_{\text {ref }, l} * \Lambda$ with $R_{\text {ref }, l}:=\left.R_{\text {ref }}\right|_{V_{\infty}=V_{\infty l \delta}}$ is usually more preferable.

The following two propositions reduce a study of the $2 \mathrm{D}$ case to the $1 \mathrm{D}$ one and, in particular, allow to apply the results of Section 3. 
Proposition 4.10. For an operator $\mathcal{S}$ admitting representation (4.27), $2 D$ condition (4.8) or (4.24) is equivalent to the collection of the respective $1 D$ conditions (2.9) or (2.30) (with the same $\hat{E} \in N D\left(\bar{\omega}^{\tau}\right)$ ) for $\mathcal{S}=\mathcal{S}_{l}, 1 \leqslant l \leqslant K-1$.

Proof. Exploiting the Parseval equality for $\mathcal{F}$ and property (4.26), we get

$$
\begin{aligned}
\left(\mathcal{S} \Phi, \bar{s}_{t} \Phi\right)_{\omega_{\delta} \times \omega_{M}^{\tau}} & =\sum_{m=1}^{M} \sum_{l=1}^{K-1}\left(\mathcal{F} \mathcal{S}^{m} \boldsymbol{\Phi}^{m}\right)^{(l)}\left(\bar{s}_{t} \mathcal{F} \Phi^{m(l)}\right)^{*} \tau_{m} \\
& =\sum_{l=1}^{K-1}\left(\mathcal{S}_{l} \mathcal{F} \Phi^{(l)}, \bar{s}_{t} \mathcal{F} \Phi^{(l)}\right)_{\omega_{M}^{\tau}}
\end{aligned}
$$

This proves the result in the case of condition (4.8) due to arbitrariness of $\mathcal{F} \Phi^{(l)}$, $1 \leqslant l \leqslant K-1$. (For $\mathcal{S}=\mathcal{S}_{\text {ref }}$, this argument has been already applied in the proof of Proposition 4.3 in Part I.)

The case of condition (4.24) is quite similar.

Proposition 4.11. For an operator $\mathcal{S}$ admitting representation (4.27), the equality holds

$$
\|\mathcal{S}\|_{M, \mathcal{E}, p, p_{1}}=\max _{1 \leqslant l \leqslant K-1}\left\|\mathcal{S}_{l}\right\|_{M, \mathcal{E}, p, p_{1}}
$$

provided that $1 \leqslant p \leqslant 2 \leqslant p_{1} \leqslant \infty$; in particular, $\|\mathcal{S}\|_{M, \mathcal{E}}=\max _{1 \leqslant l \leqslant K-1}\left\|\mathcal{S}_{l}\right\|_{M, \mathcal{E}}$.

Proof. By the Parseval equality for $\mathcal{F}$ and property (4.26), we get

$$
\|\mathcal{S} \Phi\|_{\omega_{\delta}}^{2}=\sum_{l=1}^{K-1}\left|(\mathcal{F} \mathcal{S} \boldsymbol{\Phi})^{(l)}\right|^{2}=\sum_{l=1}^{K-1}\left|\mathcal{S}_{l} \mathcal{F} \boldsymbol{\Phi}^{(l)}\right|^{2}, \quad\|\Phi\|_{\omega_{\delta}}^{2}=\sum_{l=1}^{K-1}\left|\mathcal{F} \Phi^{(l)}\right|^{2}
$$

Consequently, recalling definition (4.25), we find

$$
\|\mathcal{S}\|_{M, \mathcal{E}, p, p_{1}}=\sup _{\Lambda_{1}, \ldots, \Lambda_{K-1}} \frac{\left\|\frac{1}{\mathcal{E}}\left[\sum_{l=1}^{K-1}\left|\mathcal{S}_{l} \boldsymbol{\Lambda}_{l}\right|^{2}\right]^{1 / 2}\right\|_{L^{p_{1}\left(\omega_{M}^{\tau}\right)}}}{\left\|\frac{1}{\mathcal{E}}\left[\sum_{l=1}^{K-1}\left|\Lambda_{l}\right|^{2}\right]^{1 / 2}\right\|_{L^{p}\left(\omega_{M}^{\tau}\right)}}
$$

where supremum is taken over all $\Lambda_{1}, \ldots, \Lambda_{K-1}: \omega_{M}^{\tau} \rightarrow \mathbb{C},\left|\Lambda_{1}\right|+\cdots+\left|\Lambda_{K-1}\right| \not \equiv 0$.

Choosing all the functions $\Lambda_{1}, \ldots, \Lambda_{K-1}$ to be zero except one of them, we obtain

$$
\|\mathcal{S}\|_{M, \mathcal{E}, p, p_{1}} \geqslant \max _{1 \leqslant l \leqslant K-1}\left\|\mathcal{S}_{l}\right\|_{M, \mathcal{E}, p, p_{1}}
$$

for any $p, p_{1} \in[1, \infty]$.

On the other hand, for $1 \leqslant p \leqslant 2 \leqslant p_{1} \leqslant \infty$, by applying the generalized Minkowski inequality for sums, we get

$$
\|\mathcal{S}\|_{M, \mathcal{E}, p, p_{1}} \leqslant \sup _{\Lambda_{1}, \ldots, \Lambda_{K-1}} \frac{\left[\sum_{l=1}^{K-1}\left\|\frac{1}{\mathcal{E}} \mathcal{S}_{l} \boldsymbol{\Lambda}_{l}\right\|_{L^{p_{1}}\left(\omega_{M}^{\tau}\right)}^{2}\right]^{1 / 2}}{\left[\sum_{l=1}^{K-1}\left\|\frac{1}{\mathcal{E}} \Lambda_{l}\right\|_{L^{p}\left(\omega_{M}^{\tau}\right)}^{2}\right]^{1 / 2}}
$$

and since clearly

$$
\left\|\frac{1}{\mathcal{E}} \mathcal{S}_{l} \boldsymbol{\Lambda}_{l}\right\|_{L^{p_{1}\left(\omega_{M}^{\tau}\right)}} \leqslant \max _{1 \leqslant l \leqslant K-1}\left\|\mathcal{S}_{l}\right\|_{M, \mathcal{E}, p, p_{1}}\left\|\frac{1}{\mathcal{E}} \Lambda_{l}\right\|_{L^{p}\left(\omega_{M}^{\tau}\right)},
$$


we obtain also the inequality opposite to (4.30):

$$
\|\mathcal{S}\|_{M, \mathcal{E}, p, p_{1}} \leqslant \max _{1 \leqslant l \leqslant K-1}\left\|\mathcal{S}_{l}\right\|_{M, \mathcal{E}, p, p_{1}}
$$

The proof is complete.

Notice that in the case $p=p_{1}=2$ we could simply continue (4.29) as follows

$$
\|\mathcal{S}\|_{M, \mathcal{E}}=\sup _{\Lambda_{1}, \ldots, \Lambda_{K-1}} \frac{\left[\sum_{l=1}^{K-1}\left\|\frac{1}{\mathcal{E}} \mathcal{S}_{l} \boldsymbol{\Lambda}_{l}\right\|_{\omega_{M}^{\tau}}^{2}\right]^{1 / 2}}{\left[\sum_{l=1}^{K-1}\left\|\frac{1}{\mathcal{E}} \Lambda_{l}\right\|_{\omega_{M}^{\tau}}^{2}\right]^{1 / 2}},
$$

which more easily leads to the result.

One can easily generalize the results of Part I and this part for the problem in a rectangular parallelepiped of any dimension unbounded in one of the coordinates in one or both its directions.

Acknowledgments. The results of the paper have been mainly derived during the visit of A. Zlotnik in spring 2006 to the Département de Physique Théorique et Appliquée, CEA/DAM Ile de France (Bruyères-le-Châtel), which he thanks for hospitality. His research is also partially supported by RFBR, project nos. 07-0100416 and $05-06-80237$.

\section{REFERENCES}

[1] I. Alonso-Mallo and N. Reguera, Weak ill-posedness of spatial discretizations of absorbing boundary conditions for Schrödinger-type equations, SIAM J. Numer. Anal., 40, 134-158, 2002.

[2] X. Antoine and C. Besse, Unconditionally stable discretization schemes of non-reflecting boundary conditions for the one-dimensional Schrödinger equation, J. Comp. Phys., 188, 157$175,2003$.

[3] X. Antoine, C. Besse and V. Mouysset, Numerical schemes for the simulation of the twodimensional Schrödinger equation using non-reflecting boundary conditions, Math. Comp., 73, 1779-1999, 2004.

[4] A. Arnold, Numerically absorbing boundary conditions for quantum evolution equations, VLSI Design, 6, 313-319, 1998.

[5] A. Arnold, M. Ehrhardt and I. Sofronov, Discrete transparent boundary conditions for the Schrödinger equation: fast calculations, approximation and stability, Comm. Math. Sci., 1, 501-556, 2003.

[6] V. A. Baskakov and A. V. Popov, Implementation of transparent boundaries for numerical solution of the Schrödinger equation, Wave Motion, 14, 123-128, 1991.

[7] J.-F. Berger, M. Girod and D. Gogny, Time-dependent quantum collective dynamics applied to nuclear fission, Comp. Phys. Comm., 63, 365-374, 1991.

[8] L. Di Menza, Absorbing boundary conditions on a hypersurface for the Schrödinger equation in a half-space, Appl. Math. Letters, 9, 55-59, 1996.

[9] L. Di Menza, Transparent and absorbing boundary conditions for the Schrödinger equation in a bounded domain, Numer. Funct. Anal. and Optimiz., 18, 759-775, 1997.

[10] B. Ducomet and A. Zlotnik, On stability of the Crank-Nicolson scheme with approximate transparent boundary conditions for the Schrödinger equation, part I, Comm. Math. Sci., 4, 741-766, 2006.

[11] R. E. Edwards, Fourier Series, A Modern Introduction, Springer: New York, Heidelberg, Berlin, 2, 1982.

[12] M. Ehrhardt and A. Arnold, Discrete transparent boundary conditions for the Schrödinger equation, Riv. Mat. Univ. Parma, 6, 57-108, 2001.

[13] H. Goutte, J.-F. Berger, P. Casoly and D. Gogny, Microscopic approach of fission dynamics applied to fragment kinetic energy and mass distribution in ${ }^{238} U$, Phys. Rev. C, 71, 024316, 1-13, 2005. 
[14] U. Grenander and G. Szegö, Toeplitz Forms and Their Applications, Univ. of California Press: Berkeley and Los Angeles, 1958.

[15] B. Mayfield, Non-local Boundary Conditions for the Schrödinger Equation, Ph.D. Thesis, University of Rhode Island, Providence, RI, 1989.

[16] N. Reguera, Analysis of a third-order absorbing boundary condition for the Schrödinger equation discretized in space, Appl. Math. Letters, 17, 181-188, 2004.

[17] N. Reguera, Stability of a class of matrices with applications to absorbing boundary conditions for Schrödinger-type equations, Appl. Math. Letters, 17, 209-215, 2004.

[18] W. Rudin, Real and Complex Analysis, 2nd ed., McGraw-Hill: New York, 1974

[19] A. Schädle, Non-reflecting boundary conditions for the two-dimensional Schrödinger equation, Wave Motion, 35, 181-188, 2002.

[20] A. Schädle, Ein Schneller Faltungsalgorithmus Für Nichtrelektierende Randbedingungen, Ph.D. Thesis, Universität Tübingen, 2002.

[21] F. Schmidt and D. Yevick, Discrete transparent boundary conditions for Schrödinger-type equations, J. Comp. Phys., 134, 96-107, 1997.

[22] A. A. Zlotnik, Some finite-element and finite-difference methods for solving mathematical physics problems with non-smooth data in n-dimensional cube, Sov. J. Numer. Anal. Math. Modelling, 6, 421-451, 1991.

[23] A. A. Zlotnik and B. Ducomet, Stability of the symmetric finite-difference scheme with approximate transparent boundary conditions for the time-dependent Schrödinger equation, Dokl. Math., 75, 260-265, 2007. 